\title{
Disruption of Src Is Associated with Phenotypes Related to Williams-Beuren Syndrome and Altered Cellular Localization of TFII-I ${ }^{1,2}$
}

\author{
Laleh Sinai, ${ }^{1,2}$ Evgueni A. Ivakine, ${ }^{3}$ Emily Lam, ${ }^{1}$ Marielle Deurloo, ${ }^{4}$ Joana Dida, ${ }^{5}$ Ralph A. Zirngibl, ${ }^{6}$ \\ Cynthia Jung, ${ }^{3}$ (1) Jane E. Aubin, ${ }^{6}$ Zhong-Ping Feng, ${ }^{4}$ John Yeomans, ${ }^{5}$ Roderick R. Mclnnes, ${ }^{3,6,7}$ Lucy \\ R. Osborne, ${ }^{1,6,8}$ and John C. Roder ${ }^{2,6}$
}

DOI:http://dx.doi.org/10.1523/ENEURO.0016-14.2015

${ }^{1}$ Institute of Medical Science, University of Toronto, Toronto, Ontario, M5S 1A8, Canada, ${ }^{2}$ Lunenfeld-Tanenbaum Research Institute, Mount Sinai Hospital, Toronto Ontario, M5S 3E1, Canada, ${ }^{3}$ Programs in Genomics and Developmental Biology, The Hospital for Sick Children Research Institute, Peter Gilgan Center for Research and Learning, Toronto, Ontario, M5G 0A4, Canada, ${ }^{4}$ Department of Physiology, University of Toronto, Toronto, Ontario, M5S 1A8, ${ }^{5}$ Department of Psychology, Center for Biological Timing and Cognition, University of Toronto, Toronto, Ontario, M5S 3G3, Canada, ${ }^{6}$ Department of Molecular Genetics, University of Toronto, Toronto, Ontario, M5S 1A8, Canada, ${ }^{7}$ Lady Davis Institute, Jewish General Hospital, McGill University, Montreal, Quebec, H3T 1E2, Canada, and ${ }^{8}$ Department of Medicine, University of Toronto, Toronto, Ontario, M5S 1A8, Canada

\begin{abstract}
Src is a nonreceptor protein tyrosine kinase that is expressed widely throughout the central nervous system and is involved in diverse biological functions. Mice homozygous for a spontaneous mutation in $\mathrm{Src}\left(\mathrm{Src}^{\text {thl/thl}}\right)$ exhibited hypersociability and hyperactivity along with impairments in visuospatial, amygdala-dependent, and motor learning as well as an increased startle response to loud tones. The phenotype of Src ${ }^{\text {thl/thl }}$ mice showed significant overlap with Williams-Beuren syndrome (WBS), a disorder caused by the deletion of several genes, including General Transcription Factor 2-I (GTF2I). Src phosphorylation regulates the movement of GTF2I protein (TFII-I) between the nucleus, where it is a transcriptional activator, and the cytoplasm, where it regulates trafficking of transient receptor potential cation channel, subfamily C, member 3 (TRPC3) subunits to the plasma membrane. Here, we demonstrate altered cellular localization of both TFII-I and TRPC3 in the Src mutants, suggesting that disruption of Src can phenocopy behavioral phenotypes observed in WBS through its regulation of TFII-I.
\end{abstract}

Key words: calcium channel; general transcription factor 2 I; mouse behavior; Src tyrosine kinase; WilliamsBeuren syndrome

\section{Significance Statement}

Src tyrosine kinase phosphorylates the general transcription factor protein TFII-I, which is deleted in the neurodevelopmental disorder Williams-Beuren syndrome (WBS). We identified phenotypes such as increased sociability, visuospatial deficits, craniofacial abnormalities, and hyperactivity that overlap with symptoms of WBS in a mouse with disruption of Src. We also demonstrated altered cellular localization of TFII-I and of the TRPC3 calcium channel subunit, which is regulated by TFII-I. The finding that the Src null mouse phenocopies some features of WBS confirms its crucial role in the development and function of the nervous system, possibly through mediating changes in the cellular localization and function of TRPC3, and leading to altered agonist-induced calcium entry. 


\section{Introduction}

Src tyrosine kinases mediate a broad spectrum of physiological responses, including cell cycle control, proliferation, differentiation, migration, and survival, when activated in response to signals from diverse cellular receptors and extracellular stimuli (Thomas and Brugge, 1997). Src is highly expressed in the mammalian CNS and is involved in neuronal differentiation and neurite outgrowth (S.S. Kuo et al, 1997; W.L. Kuo et al, 1997; Hoffman-Kim et al, 2002). The NMDA receptor has been shown to be modulated by Src, and NMDA subunit phosphorylation and dephosphorylation is crucial for synaptic plasticity through the enhancement and suppression of NMDA receptor-mediated synaptic currents (Wang and Salter, 1994; Lu et al, 1998). The Src family kinases are also involved in platelet-derived growth factor and epidermal growth factor signaling (Kilkenny et al, 2003).

Src phosphorylates many downstream targets, one of which is General Transcription Factor 2-I (TFII-I), which undergoes phosphorylation-dependent shuttling between the nucleus and cytoplasm (Cheriyath et al, 2002). The GTF2I gene is commonly deleted in Williams-Beuren syndrome (WBS) (Merla et al, 2010), a neurodevelopmental disorder characterized by craniofacial dysmorphology, intellectual disability, deficits in visuospatial construction, relative strength in concrete language, social disinhibition, and nonsocial anxiety (Mervis and John, 2010). TFII-I has been described as a transcriptional regulator when in the nucleus (Roy et al, 1991), but acts as a regulator of agonist-induced calcium entry when in the cytoplasm through its competition with transient receptor potential cation channel, subfamily C, member 3 (TRPC3) for binding to phospholipase C-gamma (PLC- $\gamma$ ) (Caraveo et al, 2006).

The association of the Src pathway with a disorder involving altered social behavior suggests that Src itself may also be important for appropriate social interaction. Although Src knock-out mice have been generated previously, no behavioral assessments have been performed (Soriano et al, 1991; Kim et al, 2005). Here, we have characterized mice with a spontaneous null Src mutation for social and cognitive paradigms relevant to neurobe-

Received September 4, 2014; accepted March 2, 2015; First published March 06, 2015

${ }^{1}$ Author contributions: L.S., M.D., J.E.A., Z.-P.F., J.Y., R.R.M., L.R.O., and J.C.R. designed research; L.S., E.A.I., E.L., M.D., J.D., R.A.Z., and C.J. performed research; L.S., E.L., M.D., J.D., R.A.Z., J.E.A., Z.-P.F., J.Y., L.R.O., and J.C.R. analyzed data; L.S. and L.R.O. wrote the paper.

${ }^{2}$ This work was supported in part by grants from the Canadian Institutes of Health Research to J.E.A. (FRN69198) and L.R.O. (MOP77720).

We thank Sylvia Wong for measurements included in Table 1.

Correspondence should be addressed to either of the following: Lucy R. Osborne, Departments of Medicine and Molecular Genetics, 7360, Medical Sciences Building, 1 King's College Circle, University of Toronto, Toronto, ON, M5S 1A8, Canada, E-mail: lucy.osborne@utoronto.ca; or Laleh Sinai, Lunenfeld-Tanenbaum Research Institute, Mount Sinai Hospital, 600 University Avenue, Toronto ON, M5S 3E1, Canada, E-mail: sinai.laleh@gmail.com. DOI:http://dx.doi.org/10.1523/ENEURO.0016-14.2015

Copyright (C) 2015 Sinal et al.

This is an open-access article distributed under the terms of the Creative Commons Attribution 4.0 International, which permits unrestricted use, distribution and reproduction in any medium provided that the original work is properly attributed. havioral syndromes such as WBS. We have identified increased social interaction and recognition with deficits in learning and memory, accompanied by alterations in downstream components of the Src pathway that are implicated in the etiology of WBS. Based on these findings, we propose that Src may be a connecting bridge between neurodevelopmental disorders with aberrant social behavior.

\section{Materials and Methods}

\section{Identification of Src ${ }^{\text {thl/thl }}$ mice}

Affected mice were discovered during a routine observation of our animal colony. They were significantly smaller than their wild-type (WT) littermates and lacked incisors and some molar teeth. When maintained on a special mashed food diet, $\mathrm{Src}^{\text {thl/thl }}$ mice survived until at least 1 year of age (end of the observation period). Intercross of heterozygous $\mathrm{Src}^{\text {thl/ }}$ mice produced affected homozygous progeny at the expected Mendelian ratio.

Genome-wide mapping studies were performed on 20 affected mice using a panel of microsatellite markers positioned approximately $20 \mathrm{cM}$ apart. For narrowing down the critical interval, both microsatellite and single nucleotide polymorphism markers were used. All markers and their corresponding positions, according to the Ensembl database, are listed in Figure 1A. Genotyping was performed at The Center for Applied Genomics, Toronto. After placing the Src gene within the critical interval, we sequenced the Src coding region from one affected mouse and confirmed the Src ${ }^{\text {thl }}$ mutation.

All experiments were carried out with $S r c^{\text {thl/thl }}$ and WT littermates. Animals were housed in groups of four or five per home cage and kept on a $12 \mathrm{~h}$ light/dark cycle, with food and water available ad libitum.

\section{Genotyping}

Genotyping was performed by amplification of a $180 \mathrm{bp}$ PCR product using the primer pair: 5'-CTATCCTTCTATCAGGAATAACCAG-3' and 5'-GTTCTCCCCTACTAGGATATTG-3', followed by digestion with the Hpa II restriction enzyme (Fermentas). PCR products from mice carrying the thl mutation gained a Hpa II restriction site that resulted in the $180 \mathrm{bp}$ fragment being cleaved into four fragments (89 bp, $38 \mathrm{bp}, 35 \mathrm{bp}$, and $18 \mathrm{bp}$ ) instead of three (107 bp, $38 \mathrm{bp}$, and $35 \mathrm{bp}$ ).

\section{Western blot analysis of Src}

Protein lysates were prepared by homogenization of rapidly dissected whole adult brain in lysis buffer $(10 \mathrm{mM}$ TRIS-HCl 6.8, 2.5\% SDS, 2 mM EDTA) containing protease and phosphatase inhibitor cocktails (SigmaAldrich). Lysates were adjusted to $1 \mathrm{mg} / \mathrm{ml}$, and $50 \mu \mathrm{g}$ of protein was used for SDS-PAGE (10\% acrylamide gel) analysis and Western blotting. Western blots were probed with antibody directed against Src (Ab7950, Abcam). $\beta$-tubulin (Sigma-Aldrich) was used for loading control and protein signals were quantified using ImageJ $(\mathrm{NIH}$, http://rsb.info.nih.gov/ij). 


\begin{tabular}{|c|c|c|c|c|c|c|c|c|c|c|c|}
\hline Marker & $\begin{array}{l}\text { Start } \\
\text { position, bp } \\
\text { (ENSEMBL) }\end{array}$ & $\begin{array}{c}\text { thl } \\
1\end{array}$ & $\begin{array}{c}t h l \\
2\end{array}$ & $\begin{array}{c}t h l \\
3\end{array}$ & $\begin{array}{c}t h l \\
4\end{array}$ & $\begin{array}{c}t h l \\
5\end{array}$ & $\begin{array}{c}t h l \\
6\end{array}$ & $\begin{array}{c}t h l \\
7\end{array}$ & $\begin{array}{c}\text { thl } \\
8\end{array}$ & $\begin{array}{c}\text { thl } \\
9\end{array}$ & $\begin{array}{l}\text { thl } \\
10\end{array}$ \\
\hline D2Mit395 & $119,524,913$ & S & $\mathrm{S}$ & S & S & $\mathrm{H}$ & $\mathrm{H}$ & S & S & S & $\mathrm{S}$ \\
\hline D2Mit285 & $152,857,301$ & S & $S$ & S & $S$ & $S$ & $\mathrm{H}$ & S & S & S & $S$ \\
\hline Src & $157,418,444$ & & & & & & & & & & \\
\hline D2Mit411 & $159,586,427$ & S & $\mathrm{S}$ & S & S & $S$ & S & S & S & $\mathrm{S}$ & $\mathrm{S}$ \\
\hline rs28247368 & $159,709,338$ & $\mathrm{~S}$ & $\mathrm{~S}$ & $\mathrm{~S}$ & $\mathrm{~S}$ & $\mathrm{~S}$ & $\mathrm{~S}$ & NT & NT & NT & NT \\
\hline rs27332130 & $161,510,193$ & S & $\mathrm{S}$ & $\mathrm{S}$ & $\mathrm{H}$ & $S$ & $S$ & NT & NT & NT & NT \\
\hline rs8260591 & $163,735,302$ & $S$ & $S$ & $\mathrm{H}$ & $\mathrm{H}$ & $S$ & $S$ & NT & NT & NT & NT \\
\hline rs13476925 & $173,274,692$ & $\mathrm{H}$ & $\mathrm{H}$ & $\mathrm{H}$ & $\mathrm{H}$ & $S$ & S & NT & NT & NT & NT \\
\hline D2Mit148 & $178,800,545$ & $\mathrm{H}$ & $\mathrm{H}$ & $\mathrm{H}$ & $\mathrm{H}$ & $S$ & $S$ & $\mathrm{~S}$ & $S$ & $\mathrm{~S}$ & $\mathrm{~S}$ \\
\hline
\end{tabular}

B

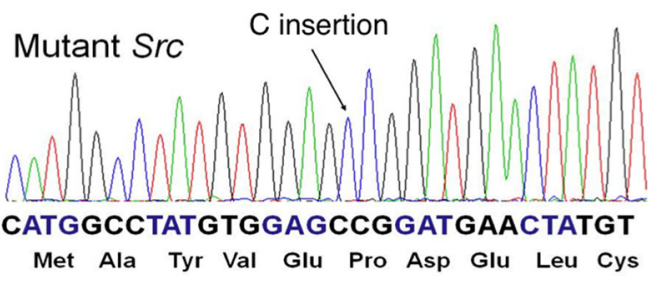

WT SrC

CATGGCCTATGTGgAgCggatgAACTATgtg

Met Ala Tyr Val Glu Arg Met Asn Tyr Val

D

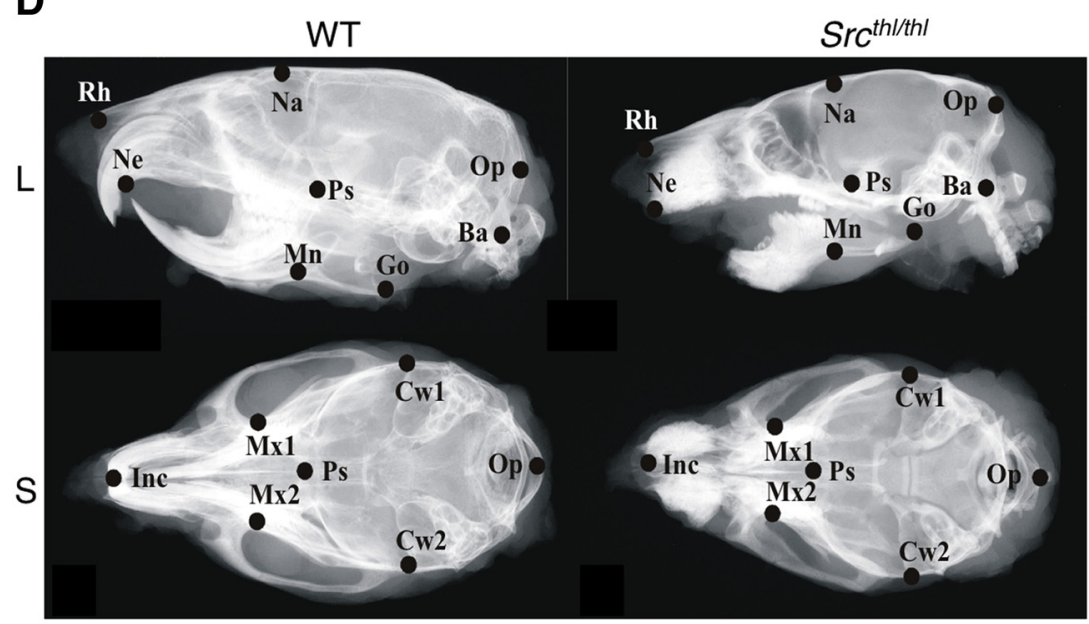

WT $S r c^{+/ t h l} S r c^{\text {thllthl }}$

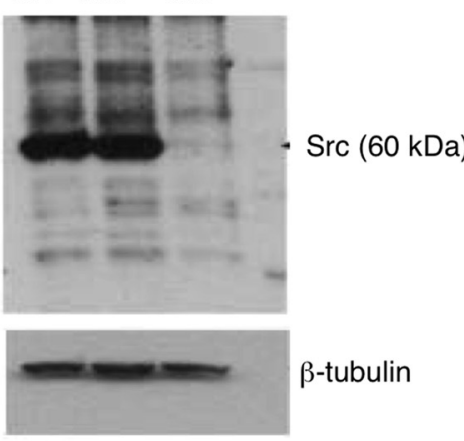

E

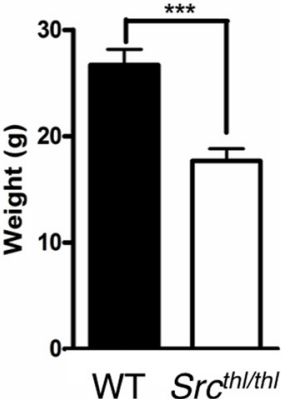

Figure 1. Craniofacial dysmorphology and growth retardation in Src ${ }^{\text {thl/thl }}$ mice. $\boldsymbol{A}$, Whole genome scan in (S129 x C57BL/6) F2 toothless mice demonstrated linkage to D2Mit411. Additional markers were used to refine the critical interval (highlighted in yellow). S, Homozygous for S129 allele; H, heterozygous for S129 and C57BL6 alleles; NT, not tested. B, Mutant mice have insertion of a C nucleotide in exon 12 of the Src gene. C, No Src protein was detected in immunoblot of whole-brain lysates from Src ${ }^{\text {thl/thl }}$ mice. $\boldsymbol{D}$, Faxitron images of WT and Src ${ }^{\text {thl/thl }}$ mouse skulls in lateral (L) and superior (S) views with landmarks used for cephalometric analysis indicated and described in Materials and Methods. Abbreviations are defined in Table 1's footnote. Analysis of the data is summarized in Table 1. $\boldsymbol{E}$, Weight of WT and Src ${ }^{\text {thl/thl }}$ mice shown as mean \pm SEM $\left(n=10 \mathrm{WT}, n=9 \mathrm{Src}^{\text {thl/thl} /}\right)$. 


\section{Bone mineral density and craniofacial analysis}

Nine-week-old male mice (four mice per genotype) were dissected to obtain the skull for faxitron analysis and the femurs and tibiae for bone mineral density determination by PIXImus densitometry (GE Medical Systems). Faxitron images were printed at the same magnification and the distances between established landmarks measured (Kiliardis et al, 1985; Lightfoot and German, 1998).

\section{Behavioral testing cohorts}

Seven different cohorts of animals were used for behavioral testing. Where cohorts underwent two different kinds of testing procedure, there was a 2 week interval between the tests. The tests were as follows: Cohort 1, olfactory test then shock threshold; Cohort 2, open field then motor function; Cohort 3, elevated plus maze then fear conditioning; Cohort 4, sociability and social novelty then novel object recognition; Cohort 5, acoustic startle response then Morris water maze; Cohort 6, ultrasonic vocalization; Cohort 7 bone mineral density and craniofacial analysis. In Cohort 4, 2 weeks separated each social test (direct social interaction, three-chamber test, tube test).

\section{Sociability and social novelty recognition}

Social approach and social novelty

We used the social approach task to assess the tendency to spend time with another mouse versus a novel object (an empty wire cage) and the ability to discriminate and choose between a familiar and new mouse. The apparatus consisted of a clear Plexiglas box $(53 \mathrm{~cm} \times 25.6 \mathrm{~cm} \times$ $23 \mathrm{~cm}$ ) divided into three chambers (outer chambers 19.5 $\mathrm{cm}$ in length; central chamber $13 \mathrm{~cm}$ in length). The outer chambers were divided from the central chamber by clear Plexiglas partitions $(11 \mathrm{~cm} \times 23 \mathrm{~cm})$ containing an opening $(7.3 \mathrm{~cm} \times 7.3 \mathrm{~cm})$. An empty wire cage (Galaxy Cup, Spectrum Diversified Designs) was used to hold the stranger mouse to prevent aggressive interactions and also support the availability of visual, auditory and olfactory cues. The wire cage was $11 \mathrm{~cm}$ in height, with a bottom diameter of $10.5 \mathrm{~cm}$ and bars spaced $1 \mathrm{~cm}$ apart. A beaker full of water was placed on the top of the cage to prevent it from moving. The cage was located in the center of each outer chamber throughout the experiment to permit social investigation.

The test was carried out according to previously published procedures (Nadler et al, 2004), on 8- to 10-weekold littermates with a habituation period followed by a 10 min experimental trial, and the unfamiliar mouse (Mouse 1) was alternated between right and left side chambers across subjects. Time spent in each chamber, number of entries, and time sniffing each wire cage was measured by an observer using Noldus software. In a subsequent 10 min trial, a new unfamiliar mouse was placed in the wire cage that had been empty during the previous session. The test mouse could choose between the first familiar mouse (Mouse 1) and the novel unfamiliar mouse (Mouse 2). Measures were scored as described above.

Direct social interaction test

The test mouse (male Src ${ }^{\text {thl/thl }}$ ) and social target mouse (weight- and age-matched unfamiliar male 129Sv/
C57BL6/J) were placed in opposite corners of an unfamiliar neutral cage $(30 \mathrm{~cm} \times 17 \mathrm{~cm} \times 12 \mathrm{~cm})$, which was illuminated (280 lux) as previously described (File et al, 2001). Neutral cages were changed between experiments for each tested pair and each social target mouse was used only once. Scoring behavior parameters started with the first interaction and lasted for $5 \mathrm{~min}$. During this time, social investigation and nonsocial behavior such as selfgrooming, cage exploration (sniffing around the cage), and rearing were measured. The test mouse was not habituated to the unfamiliar cage and all measures were scored only after introduction of the social target mouse.

\section{Tube test of social dominance}

The tube is an additional measure of social behavior (Shahbazian et al, 2002; Spencer et al, 2005). In this test, 8- to 10-week-old WT and Src ${ }^{\text {thl/thl }}$ littermates were placed in opposite ends of a clear acrylic cylindrical tube ( $3.5 \mathrm{~cm}$ diameter and $33 \mathrm{~cm}$ in length) and released at the same time. When they entered the tube, the mouse that backed out of the tube first was considered the loser.

\section{Olfactory test}

Olfactory function was measured by modifying the olfactory habituation/dishabituation task, as previously described (Wrenn et al., 2003). The mice were habituated to a novel clean testing cage for $5 \mathrm{~min}$. Mice were then tested for time spent sniffing a Q-Tip suspended from the cage lid. The $\mathrm{Q}$-Tip contained two different flavors for the habituation phase (almond extract; McCormick; 1:100 dilution) and dishabituation phase (lemon extract; McCormick; 1:100 dilution). A sequence of six trials for the almond extract assayed habituation to the same smell. Switching to the lemon smell on the Q-Tip assayed dishabituation (recognition that an odor is new). Each swab was presented for a 2 min period for six trials for the almond extract and one trial for the lemon extract. The time spent sniffing the Q-Tip was measured with a stopwatch by an observer. Sniffing was scored when the nose was within $2 \mathrm{~cm}$ of the Q-Tip.

\section{Ultrasonic vocalization}

Ultrasonic vocalization (USV) recordings were carried out on mice between 11 and 16 weeks of age. In the male-female paradigm, mating-induced USVs and video recordings were obtained as follows. USVs were obtained with a D1000X ultrasound recorder (Pettersson Elektronik AB) for $5 \mathrm{~min}$ at a sampling frequency of $250 \mathrm{kHz}$. The microphone was suspended from a Plexiglas chamber put on top of the mating cage. Spectrographs (20-125 kHz) were generated by discrete Fourier transformation (256 bins) and analyzed with Avisoft SASLab Pro Software v4.39 (Avisoft Bioacoustics). A Vostro 1710 laptop running Windows XP was used for analysis of sonograms by a trained observer who was blind to genotype.

The female mouse was removed from the home cage and placed in a clean standard polyethylene cage $(22 \mathrm{~cm}$ $\times 30 \mathrm{~cm} \times 15 \mathrm{~cm}$ ) with fresh bedding. The clean cage was brought into the testing room adjacent to the housing room, illuminated by a 40 watt red bulb. The top of the cage was removed and replaced with the Mating Chamber Addition, which consisted of four polyethylene exten- 
sion walls with an open top and with an arm suspended in the center of the chamber. The microphone was suspended in the center of the cage, $12 \mathrm{~cm}$ above the floor. A video camera outside the lateral wall was positioned to record behaviors synchronously for further analysis. WT females were paired with either WT or Src ${ }^{\text {thl/thl }}$ males. The female was left to habituate to the testing room for $5 \mathrm{~min}$ (Gourbal et al, 2004; Hoffman et al, 2012) to reduce stress and focus the attention of the female on the introduced male, thus increasing the probability of vocalization (Zampieri et al, 2014). The male was then introduced to the cage and pairing was allowed to continue for $5 \mathrm{~min}$. Audio and video recordings were obtained for the entire duration of the pairing.

In our female-female social reunion paradigm, samelitter females were reunited following a period of separation. WT females were housed in separate home cages from $\mathrm{Src}^{\text {thl/thl }}$ females due to competition for food, so female-female reunions were always between mice of the same genotype. The day prior to testing, one mouse was removed from the home cage and put into a clean standard polyethylene host cage. Twenty hours following separation, the host cage was taken to the testing room and the host was left to habituate for several minutes without food, water, or nesting to minimize distraction. The top of the cage was removed and replaced with the Mating Chamber Addition for the same purpose as in the male-female paradigm. Following habituation, a mouse from the home cage, "the visitor", was introduced into the host cage and the reunion was allowed for $5 \mathrm{~min}$. USVs and video recordings were obtained for the entire trial. Total USV counts generated each minute were counted and grouped into three categories [Type 1, flat; Type 2, broken; Type 3, frequency modulated, as previously described (Wang et al, 2008)] for the entire trial. The total number of calls and type of calls within each minute were scored.

\section{Exploratory activity and anxiety}

Motor activity of 6-week-old mice was measured in the open field, and anxiety of 8-week-old mice was assessed in the elevated plus maze. Anxiety-like behaviors were measured as time spent in the center of the open field and as time spent in the open arms of an elevated plus maze according to previously published procedures (Kirshenbaum et al, 2011). Briefly, in the open field, mice were placed in the center of a transparent Plexiglas arena $(41.25 \mathrm{~cm} \times 41.25 \mathrm{~cm} \times 31.25 \mathrm{~cm})$ illuminated by 200 lux, and total distance travelled, time spent in the center (31 $\mathrm{cm} \times 31 \mathrm{~cm})$ and rearing were recorded for $30 \mathrm{~min}$. The elevated plus maze consisted of a central platform $(5 \mathrm{~cm}$ $\times 5 \mathrm{~cm})$ with two opposing open arms $(25 \mathrm{~cm} \times 5 \mathrm{~cm})$ and two opposing arms enclosed by opaque Plexiglas walls $(25 \mathrm{~cm} \times 5 \mathrm{~cm} \times 30 \mathrm{~cm})$. All arms were made of opaque Plexiglas and the entire apparatus was elevated $50 \mathrm{~cm}$ from the floor. Experiments were conducted in a dark room and open arms were brightly illuminated (700 lux). Mice were placed on the central platform facing a closed arm and the number of entries into each arm and duration in each arm was scored for $5 \mathrm{~min}$. Time spent in the center of the maze was not recorded.

\section{Sensory and motor function}

All tests of motor performance were carried out on 8- to 10 -week-old littermates.

\section{Rotarod}

Mice were tested for two trials per day with a $60 \mathrm{~min}$ intertrial interval for 4 consecutive days. We used an Economex Rotarod apparatus (Columbus Instruments). The $3 \mathrm{~cm}$ ribbed plastic rotating axle was hung at a height of $30 \mathrm{~cm}$ above the base of the apparatus. Mice were placed on top of the rod, facing away from the experimenter. In this orientation, forward locomotion opposite to rotation of the rod is necessary to avoid falling. During the stationary habituation session, each mouse was first observed for $60 \mathrm{~s}$ without any rotation to allow the animals to become accustomed to the apparatus. The axle was then adjusted for a fixed constant motor speed of 5 rotations per minute (rpm) and each mouse observed for a total of $90 \mathrm{~s}$. Next, beginning at $5 \mathrm{rpm}$, the rotation was accelerated in increments of $0.1 \mathrm{rpm} / \mathrm{s}$ and the latency to fall off the axle was recorded for each mouse for a maximum period of $5 \mathrm{~min}$. Average time spent on the rotarod was calculated for each day.

\section{Grip strength}

The maximal muscle strength of the forelimbs, and both forelimbs and hindlimbs together was measured with an isometric transducer attached to a $3 \mathrm{~mm}$ diameter metal bar (Ugo Basile). For the forelimb measurements, each mouse gripped the bar with its forelimbs and was then slowly pulled backwards until it released the metal bar. The transducer measured the maximal grip strength in grams. Five trials were performed in each testing session, and the mean value was calculated. For the hindlimb measurements, a similar method was used but each mouse was allowed to grab with both its front and hind paws. The forelimb measurement was subtracted from the combined forelimb and hindlimb measurement to obtain the hindlimb grip strength.

\section{Balance beam test}

Mice were acclimated to a round, 30-cm-long, 3-mmwide beam elevated $28 \mathrm{~cm}$ above a padded base. A $60 \mathrm{~W}$ lamp at the starting platform served as an aversive stimulus and the opposite end of the beam was in a darkened escape box on the arriving platform. Traversal time and number of slips were measured as mice travelled across the beam. All testing was performed in triplicate and mean values were used for subsequent statistical analyses.

\section{Acoustic startle response}

The acoustic startle response test was carried out on 10-week-old littermates. Each mouse was placed into the startle chamber and allowed to acclimatize for $15 \mathrm{~min}$. The mouse was then presented with $25 \mathrm{~ms}$ startle stimuli of varying intensities (70-120 dB), with an interstimulus interval of 25-30 s. Startle stimuli were presented in three blocks each composed of two presentations of the 11 stimulus intensities given in a pseudorandom order. The 
average startle amplitude for each stimulus intensity was calculated from the three blocks.

\section{Learning and memory tasks}

Morris water maze

The Morris water maze (MWM) was used to test spatial memory. Prior to the test, mice were handled for 2 min per day for 7 consecutive days. Testing was carried out on mice between 11 and 16 weeks of age. Mice were given two trials per day, with a $30 \mathrm{~s}$ intertrial interval for 6 consecutive days. This challenging approach was used to enable us to detect even subtle deficits in visuopatial learning and memory. Two hours after the training on day 6 , a probe trial was given to test spatial learning. Mice were placed in the pool at the opposite quadrant of the platform position and were given $60 \mathrm{~s}$ to search for the platform.

The MWM consisted of a white plastic 117-cmdiameter circular pool filled with water (with constant $26{ }^{\circ} \mathrm{C}$ temperature) made opaque by white latex paint. Around the outside of the pool, 2D and 3D visual cues were displayed in a dimly lit room. The pool was divided into four equal quadrants labelled northeast, southeast, southwest, and northwest. A hidden platform $(10 \mathrm{~cm}$ diameter) submerged $1 \mathrm{~cm}$ below the water surface was placed at the center of one of the four quadrants (the target quadrant). A closed-circuit video camera was mounted directly above the center of the pool and connected to an image analyzer (HVS Image), which digitized the path data.

Mice were given two trials per day, with a $30 \mathrm{~s}$ intertrial interval for 6 consecutive days. During the visible platform trial, the platform remained submerged, but was flagged with a small spatial cue. The mouse was first placed on the platform for $30 \mathrm{~s}$, then placed in the water at a pseudorandom start position. The mouse was given a maximum of $60 \mathrm{~s}$ to find the platform, following which it was placed back onto the platform. After $30 \mathrm{~s}$ on the platform, this training procedure was repeated once more. The platform remained at the same position during all trials. The hidden platform trial was performed in the same way as the visible platform trial, but with the visual cue removed. Two hours after the training on day 6 , a probe trial was given to test spatial learning. Mice were placed in the pool at the opposite quadrant of the platform position and were then given $60 \mathrm{~s}$ to search for the platform. For the purpose of statistical analysis, crossings in the quadrant containing the target platform were compared to the averaged crossings of a similarly sized and positioned area within each of the three nontarget quadrants.

\section{Fear conditioning}

Fear conditioning was performed in a testing chamber (25 $\mathrm{cm} \times 30 \mathrm{~cm} \times 25 \mathrm{~cm}$, MED Associates) fitted with a removable grid floor of 36 stainless steel rods $(3.2 \mathrm{~mm}$ diameter, $4.7 \mathrm{~mm}$ apart) connected to a constant current shock generator, and an amplifier and speaker. A 12 inch, $8 \mathrm{~W}$ fluorescent tube (GE Lighting Canada) illuminated the chamber interior. A computer running automated fear conditioning software (FreezeFrame; Actimetrics Soft- ware) administered foot shocks and auditory tones. Video images were recorded from the chamber and the activity of subjects was recorded throughout the experiment. Immediately prior to training, the chamber was cleaned with $70 \%$ ethanol and a white cloth covered the front. Conditioning consisted of two pairings of an auditory tone with a continuous foot shock. Each mouse was placed inside the conditioning chamber for 2 min before the onset of a conditioned stimulus (CS; an $85 \mathrm{~dB}$ tone), which lasted for $30 \mathrm{~s}$. A 2 s unconditioned stimulus (US; foot shock, 0.5 $\mathrm{mA}$ ) was delivered immediately after the termination of the CS. Each mouse remained in the chamber for an additional $120 \mathrm{~s}$, followed by another CS-US pairing. The mouse was returned to its home cage after another 2.5 $\min$.

Approximately $24 \mathrm{~h}$ later, each subject was returned to the chamber and percentage of freezing was monitored for $3 \mathrm{~min}$ in the conditioned context. The activity of each subject was recorded at $0.25 \mathrm{~s}$ intervals using the FreezeFrame automated fear conditioning software (Actimetrics Software), which detects any kind of movement. The mean activity during the context exposure was calculated, the mean baseline activity subtracted, and the resulting figure used as a measure of contextual learning. Four hours later, the context was changed by covering the grid floor with a sheet of white PerspexTM (polymethyl methacrylate), inserting two sheets of transparent Perspex into the chamber to give it a prism shape, cleaning the chamber with $1 \%$ vinegar, covering the front door with a striped black and white card, and turning on the ceiling lights. Each mouse was placed into the altered chamber, and allowed 3 min for exploration in the novel environment, after which the auditory tone of 3 min duration was delivered. The mean activity during cue delivery was calculated, the mean activity in the novel context (prior to the presentation of the tone) subtracted, and the resulting figure taken as a measure of cue-associated learning.

\section{Shock threshold}

Shock threshold was assessed by delivering foot shocks starting at $0.075 \mathrm{~mA}$ and increasing by $0.05 \mathrm{~mA}$ every 30 $s$ in the conditioning chamber. The experiment was terminated at a shock intensity sufficient to induce vocalization. Testing was carried out on mice between 11 and 16 weeks of age.

\section{Visual-object and novelty recognition}

The visuospatial and novelty recognition task was carried out as previously described ( $\mathrm{Ng}$ et al, 2009). The task was performed in a transparent Plexiglas open field $(41 \mathrm{~cm} \times$ $41 \mathrm{~cm} \times 31 \mathrm{~cm}$ ) equipped with infrared beams to detect locomotor movements (model 7420/7430; Ugo Basile). Four of the objects used in this task were similar in shape, color, and material (approximately $7 \mathrm{~cm} \times 6 \mathrm{~cm} \times 6 \mathrm{~cm}$ ) for the spatial recognition task, and one object was different for the novel object recognition task. Animal behavior was recorded by an observer and analyzed using The Observer 5.0 (Noldus). On the test day, each mouse was individually placed in the center of the empty arena to habituate for $5 \mathrm{~min}$. The mouse was then placed in a holding cage for $2 \mathrm{~min}$. Two objects were placed in spe- 
cific positions in the corner of the arena and another two objects were positioned in the center of the arena. The mouse was returned to the center of the arena and allowed to explore the objects for three continuous $5 \mathrm{~min}$ sessions (training phase). Training to object exploration was measured by recording the time spent exploring the objects across the sessions. A mouse was considered to be exploring an object if its snout was in contact with the object. At the end of the training phase, the mouse was again placed in the holding cage for 2 min and the two center objects were moved to the corner positions in order to assess response to a spatial change.

The mouse was returned to the arena and the time spent exploring the displaced and nondisplaced objects was recorded for 5 min (spatial change phase). Reaction to a spatial change was assessed by calculating the ratio of the amount of time spent exploring the displaced object over the total time spent exploring the nondisplaced objects (preference index) by each mouse. Mice would be expected to spend more time exploring the displaced object than the nondisplaced objects due to the residual memory of the nondisplaced object and its position. Response to novelty was also examined. Directly after the spatial change phase, the test subject was returned to the holding cage for $2 \mathrm{~min}$, during which one of the familiar nondisplaced objects in the arena was replaced by a novel object in the same location. The mouse was returned to the center of the arena for a 5 min period. Measurements were taken as described for the previous phase, and the response to novelty change was evaluated by calculating the ratio of the amount of time spent exploring the novel object over the total time spent exploring the familiar objects (preference index) by each mouse. Mice would be expected to spend more time exploring the novel object than the familiar object due to the residual memory of the familiar object. Testing was carried out on mice between 11 and 16 weeks of age.

\section{Cellular localization of TFII-I and TRPC3 \\ Cellular fractionation}

Adult mice were killed by cervical dislocation, and brains were removed immediately. One hemisphere of the brain was finely diced and homogenized with $2 \mathrm{ml}$ homogenizing buffer (HB) containing $25 \mathrm{mM} \mathrm{KCl}, 1 \mathrm{mM} \mathrm{MgCl}_{2}, 20$ mM HEPES (pH 7.4), 1 mM EGTA, 0.2 mM DTT, $0.32 \mathrm{M}$ sucrose, $1 \mathrm{mM}$ PMSF, $1 \mathrm{mM}$ protease inhibitor cocktail (P8340; Sigma-Aldrich), and $1 \mathrm{mM}$ sodium orthovanadate. Samples were homogenized by five strokes of a Teflon homogenizer followed by an incubation of 2 min on ice in HB and then homogenized with 20 strokes of a Dounce homogenizer. All spins were performed at $4{ }^{\circ} \mathrm{C}$. The homogenate was first spun at $600 \mathrm{~g}$ for $10 \mathrm{~min}$. The pellet $(\mathrm{P} 1)$ was further treated to obtain the nuclear fraction, and the supernatant (S1) was stored at $-20^{\circ} \mathrm{C}$ for later fractionation. Resuspended in $1 \mathrm{ml} \mathrm{HB}, \mathrm{P} 1$ was passed through a $27 \mathrm{G}$ syringe and spun again at $600 \mathrm{~g}$ for $10 \mathrm{~min}$. The pellet was then resuspended in $1 \mathrm{ml}$ nuclear buffer $2.2 \mathrm{M}$ sucrose, $1 \mathrm{mM} \mathrm{MgCl}, 10 \mathrm{mM}$ HEPES, pH 7.4) and homogenized by eight strokes of the
Teflon. After spinning the homogenate at $75,000 \mathrm{~g}$ for 75 min, the resulting nuclear pellet was resuspended in RIPA buffer (10 mM Tris- $\mathrm{HCl}$, pH 8.0, $100 \mathrm{mM} \mathrm{NaCl}, 0.1 \%$ SDS, $0.5 \%$ sodium deoycholate, $1 \%$ NP- $40,1 \mathrm{mM}$ EDTA, $1 \mathrm{mM}$ protease inhibitor cocktail) and stored at $-20^{\circ} \mathrm{C}$. S1 was spun at $10,000 \mathrm{~g}$ for $15 \mathrm{~min}$, and the supernatant was further spun at $100,000 \mathrm{~g}$ for $60 \mathrm{~min}$ to obtain the cytosolic fraction (supernatant). The pellet was resuspended in $1 \mathrm{ml} \mathrm{HB}$ and spun again at 100,000 $\mathrm{g}$ for $60 \mathrm{~min}$ followed by a last wash in $0.5 \mathrm{ml} \mathrm{HB}$ at $100,000 \mathrm{~g}$ for 60 min. The pellet was then resuspended in RIPA buffer and constituted the membrane fraction.

Western blot analysis Fractions were quantified by $D_{C}$ Protein Assay (Bio-Rad), and 30-40 $\mu \mathrm{g}$ was used for Western blot analyses by SDS-PAGE on $8 \%$ acrylamide gels. After blotting onto a PVDF membrane, the nuclear fraction was probed with anti-TFII-I mouse monoclonal antibody (610943; BD Biosciences) and normalized against nuclear loading control, nucleolin (ab22758; Abcam). The membrane fraction was probed with antiTRPC3 rabbit polyclonal antibody (ab51560; Abcam) and normalized against membrane loading control, $\mathrm{N}$-cadherin (610920; BD Biosciences). ImageJ (NIH, http://rsb.info.nih.gov/ij) was used to quantify the relative band densities.

Dissociated primary cortical cultures Prenatal pups (embryonic day 17-18) produced from a Src ${ }^{\text {thl/ }+}$ intercross were used to prepare cortical cultures according to previously published protocols (Broeke et al, 2010) with modifications. All experiments were carried out with $\mathrm{Src}^{\text {thl/thl }}$ and WT littermates. Specifically, dissected cortex was digested with $0.025 \%$ Trypsin/EDTA at $37{ }^{\circ} \mathrm{C}$ in HBSS (Sigma-Aldrich) for $20 \mathrm{~min}$ and washed with prewarmed medium to stop digestion. Cells were triturated approximately 10 times with a $1000 \mu \mathrm{l}$ tip. Neurons were centrifuged at $1000 \mathrm{rpm}$ for $5 \mathrm{~min}$, supernatant was removed and cells resuspended in culture media. Cell density was determined using an Improved Neubauer hemocytometer and low-density cultures were plated on Poly-D-Lysine $(0.1 \mathrm{mg} / \mathrm{ml}$; Sigma-Aldrich) coated glass coverslips (18 $\mathrm{mm}$; \#1.5, Warner Instruments) at 100,000 neurons $/ \mathrm{cm}^{2}$. Neurons were maintained in Neurobasal medium (Invitrogen) with 2\% B27 (Invitrogen), 1× Penn/Strep (Invitrogen), and $2 \mathrm{mM}$ GlutaMAX (Invitrogen) at $37^{\circ} \mathrm{C}$ with $5 \% \mathrm{CO}_{2}$ for 6 days in vitro (DIV6).

\section{Immunocytochemistry}

Neurons at DIV6 were fixed with $4 \%$ paraformaldehyde/4\% sucrose in PBS for 20 min, washed $3 \times$ with PBS, and permeabilized with $0.1 \%$ Triton $X-100$ in PBS for $20 \mathrm{~min}$ at room temperature (RT). Cells were incubated with specific primary antibodies overnight at $4{ }^{\circ} \mathrm{C}$ : antiTRPC3 (rabbit polyclonal, 1:500; \#NB110-74935, Novus Biologicals), anti-TFII-I (goat polyclonal, 1:50; \#sc-9943 X, Santa Cruz Biotechnology), anti-NeuN (mouse monoclonal, 1:200; \#MAB377, Millipore). Cells were then incubated with their respective secondary antibody: Alexa Fluor 488 goat anti-rabbit, Alexa Fluor 568 rabbit antigoat, and Alexa Fluor 633 goat anti-mouse, (1:500; Molecular Probes) at RT for $1 \mathrm{~h}$ and washed $3 \times$ with PBS. Coverslips were then mounted on slides with ProLong 
Gold (Invitrogen) and dried in the dark at RT. When cells were incubated with secondary antibodies only, no signal was observed.

\section{Image acquisition}

Images were captured with an Olympus Confocal Laser Scanning Microscope FV10i-DOC with a $60 \times$ phase contrast oil-immersion objective (NA 1.35) and 488, 543, and 633 lasers. Each Z-plane was $0.3 \mu \mathrm{m}$. All cells were imaged at a resolution of $1024 \times 1024$ pixels using the same magnification and laser settings. Fluorescence intensity was measured in a region of interest over the cell and corrected for background measured outside of the cell. To test whether the fluorescence intensity of TRPC3 and TFII-I was significantly different between Src ${ }^{\text {thl/thl }}$ and their WT littermates, the mean amplitude fluorescence intensity (in arbitrary units) was measured from the middle section of the entire z-stack. NeuN was used as a neuronal marker. In the figures, each bar represents the average protein level fluorescence intensity from all cells measured under that condition. Fluorescence intensity was measured using ImageJ (NIH, http://rsb.info.nih.gov/ij).

\section{Statistical analysis}

One-way ANOVA (balance bean, grip strength, rotarod, acoustic startle, fear conditioning, shock threshold, visual object recognition), two-way ANOVA (social approach, open field, Morris water maze) or repeated-measures ANOVA (tube test, olfactory test, USVs) were used to test for the effect of genotype. If this was significant, the data was further analyzed using Tukey's post hoc test. Western blot band densities were analyzed using Students $t$ test and fluorescence intensity using one-way ANOVA. All figure data represents mean \pm SEM. An analysis with a value of $p<0.05$ was considered to be statistically significant.

\section{Study approval}

All animal procedures were approved by the Animal Management Committee of Mount Sinai Hospital, the Toronto Center for Phenogenomics, and the University of Toronto Animal Care Committee, and performed in accordance with the guidelines provided by the Canadian Council on Animal Care.

\section{Results}

\section{Mutation in the Src gene leads to craniofacial abnormalities}

During a routine observation of our animal colony, we noticed two 12-d-old "toothless" pups (one male and one female) that could be distinguished from their littermates by small size and a lack of incisors. Both parents lacked the observed phenotype, suggesting it was inherited as an autosomal recessive trait. These mice were on the mixed S129 genetic background derived from an R1 embryonic stem cell line. To identify the mutation responsible for this phenotype, we crossed the parents with C57BL6/J mice, intercrossed the resulting progeny, and performed genome-wide linkage analysis on 20 (S129xC57BL6/J) F2 "toothless" (thl) mice. Only one marker, D2Mit411, of S129 origin showed significant linkage with the lack of
Table 1 Linear variables of the skull in WT and Src ${ }^{\text {thl/thl }}$ mice

\begin{tabular}{llllll}
\hline Measurement & \multicolumn{2}{l}{ Wildtype } & \multicolumn{2}{l}{ Src ${ }^{\text {thl/thl }}$} & $p$ value \\
& Mean & SEM & Mean & SEM & \\
Ps-Rh & 145.1 & 1.4 & 117.6 & 4.2 & 0.0008 \\
Op-Ne & 235.5 & 1.3 & 200.8 & 5.2 & 0.0007 \\
Na-Rh & 111.3 & 1.8 & 91.0 & 3.4 & 0.0018 \\
Go-Mn & 56.5 & 3.6 & 49.9 & 3.7 & 0.24 \\
Ps-Na & 77.8 & 0.9 & 65.5 & 1.3 & 0.00024 \\
Ps-Ba & 110.5 & 2.1 & 97.5 & 2.7 & 0.0092 \\
Mx1-Mx2 & 61.0 & 0.6 & 56.0 & 2.3 & 0.077 \\
Cw1-Cw2 & 117.3 & 0.5 & 110.8 & 1.9 & 0.017 \\
Op-Inc & 261.4 & 0.7 & 221.0 & 6.9 & 0.0011 \\
Ps-Inc & 113.1 & 1.4 & 94.4 & 5.1 & 0.013 \\
\hline
\end{tabular}

Nine-week old male mice $\left(\mathrm{n}=2 \mathrm{WT}, \mathrm{n}=2 \mathrm{Src}^{\text {thl/th/}}\right)$ were dissected to obtain the skull for faxitron analysis. Images were printed at the same magnification and the distances between established landmarks measured. Data are presented as mean \pm SEM.

Lateral cephalometric landmarks: Ba: basion, the most posterior point of occipital bone anterior to the foramen magnum; Cw1 and Cw2: most lateral points of the calvarium; Go: gonion, the most posterior point of the mandibular angular process; Mn: point in the deepest part of the antegonial notch curvature; $\mathrm{M} \times 1$ and $\mathrm{M} \times 2$ : most anterior and posterior points of the maxilla; $\mathrm{Na}$ : nasion, the junction of the frontonasal and internasal sutures; Ne: nasale, intersection of nasal bones, rostral point; Ps: pre-sphenoid, the middle of the presphenoethmoidal synchrondrosis; Inc: incisor, the most prominent point between incisal edges of lower incisors.

Dorsoventral cephalometric landmarks: Rh: rhinion, the most anterior point of the nasal bones in the midline; Op: opisthion, midsaggital point on the posterior margin of the foramen magnum.

incisors trait. To identify boundaries of the critical interval region containing the mutation of interest, $10 \mathrm{thl}$ mice were genotyped for additional markers surrounding D2Mit411 (Fig. 1A). This allowed us to narrow down the position of the causative mutation to an approximately 8.5 $\mathrm{Mb}$ interval between D2Mit285 (Ensembl position: 152857301) and rs27332130 (Ensembl position: 161510193). One of the genes within the critical interval region, Src (Ensembl position: 157418444), caught our attention due to a known role of Src protein in the bone remodelling process (Lowe et al, 1993). Moreover, Srcnull mice lack incisors, similar to our th/ mutants (Tiffee et al, 1999). To evaluate whether th/ was a novel mutation in the Src gene, we sequenced the Src coding region and discovered the insertion of a $\mathrm{C}$ nucleotide into exon 12 , resulting in a frame shift and premature stop codon (Fig. $1 B$ ). Immunoblotting of brain lysates of the Src ${ }^{\text {thl/thl }}$ mice failed to detect the predicted $\sim 47 \mathrm{kDa}$ truncated Src protein (Fig. 1C), implying that the mRNA was likely degraded by nonsense-mediated decay.

In addition to their small size and the lack of incisors, $\mathrm{Src}^{\text {thl/thl }}$ mice had variable numbers of molar teeth (usually one or two were absent, sometimes from the upper jaw and sometimes from the mandible). Since visual inspection revealed that the $S r c^{\text {thl/thl }}$ mice exhibited craniofacial anomalies, faxitron images were used for cephalographic analysis (Fig. 1D). All of the parameters analyzed showed a significant reduction in length for the Srcthl/thl mice, with the exception of the Mx1-Mx2 distances, which tended to be reduced, and Go-Mn, which was not affected (Table 1). Notably, the cranial base in these mice was shortened both anteriorly and posteriorly. Defects in incisor and molar tooth formation and eruption were also evident, similar to that previously described in Src mutants (So- 
A

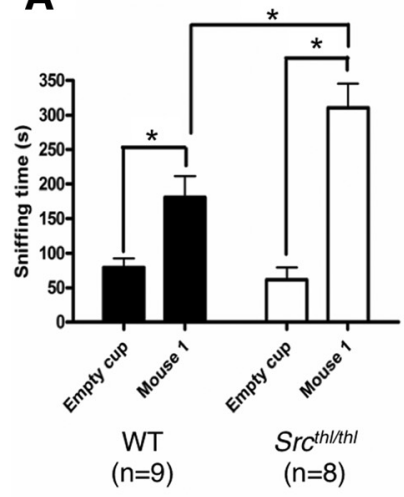

B

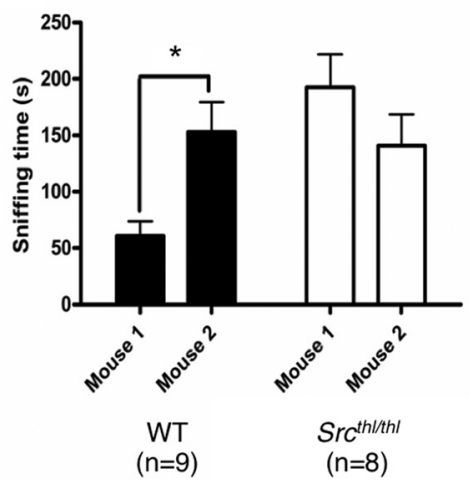

C

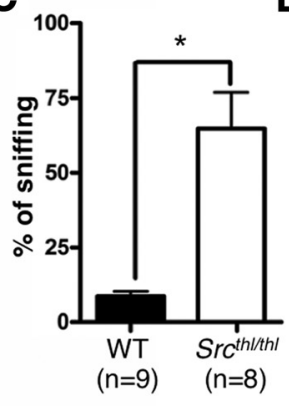

D

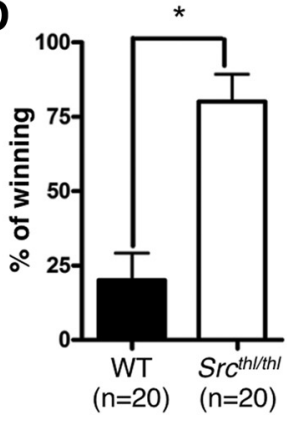

E

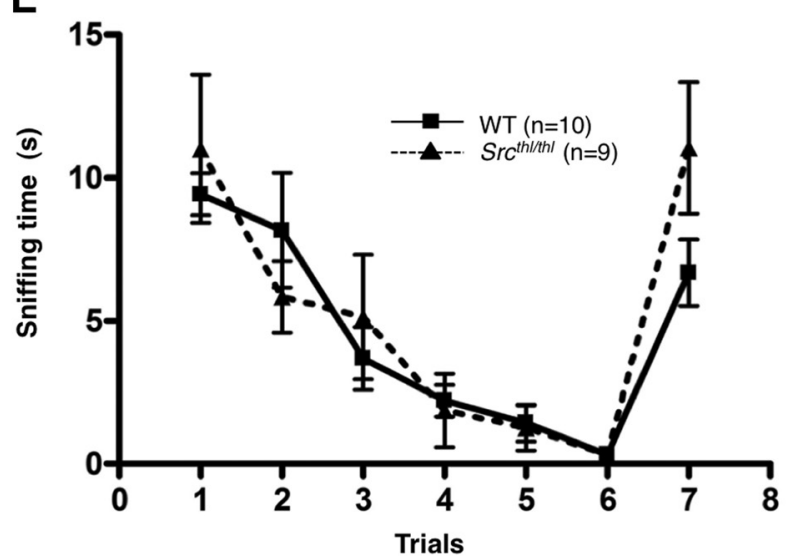

Figure 2. Social approach behaviors are increased and social recognition is impaired in Src ${ }^{\text {thl/thl }}$ mice. $\boldsymbol{A}$, Src ${ }^{\text {thl/thl }}$ mice spent more time sniffing a social cage versus a nonsocial cage ( $n=9$ WT males, $n=8 \mathrm{Src}^{\text {thl/thl }}$ males). $\boldsymbol{B}$, During the second phase of the test to measure social recognition, Src $^{\text {thl/thl }}$ mice did not show a preference for a novel mouse versus a familiar mouse from the first phase ( $n=9$ WT males, $n=8 \mathrm{Src}^{\text {thl/thl }}$ males). $* p<0.05$, compared to the chamber with empty cage, between genotypes and within genotypes, respectively. $\boldsymbol{C}$, In a direct social interaction test, Src ${ }^{\text {thl/thl }}$ mice showed an increased frequency of interactions with a stranger mouse. Data are presented as mean \pm SEM $\left(n=9\right.$ WT males, $n=8$ Src ${ }^{\text {thl/thl }}$ males $)$. $* p<0.05$, between genotypes. $\boldsymbol{D}$, Src ${ }^{\text {thl/thl }}$ mice showed social dominance over their WT littermates in a tube test ( $n=20$ WT males, $n=20$ Src ${ }^{\text {thl/thl }}$ males). $* p<0.05$, between genotypes. $\boldsymbol{E}, \mathrm{Src}^{\text {thl/thl }}$ mice were able to habituate to a smell over time and dishabituate toward a novel smell, demonstrating intact olfaction ( $n=10$ WT (5 males, 5 females), $n=9 \mathrm{Src}^{\text {thl/thl }}$ (5 males, 4 females)). Data are presented as mean \pm SEM.

riano et al, 1991; Tiffee et al, 1999). The Src ${ }^{\text {thl/thl }}$ mice were smaller in size with body weight reduction (Fig. 1E).

\section{Social interaction and social recognition is altered in Src mutant mice}

Social approach and short-term social recognition

We used a three-chambered apparatus in which mice were tested in two different trials. In the first trial, the subject mouse was given the choice to spend time with a social object (mouse) or spend time with a nonsocial object (empty). There was a statistically significant interaction between social versus nonsocial object and genotype $\left(p=0.0089, F_{(1,15)}=9.02\right)$. The effect of genotype was not quite significant $\left(p=0.052, F_{(1,15)}=4.42\right)$. The effect of social versus nonsocial object was highly significant $\left(p=0.0001, F_{(1,15)}=50.85\right)$. Both Src ${ }^{\text {thl/thl }}$ and WT mice showed a preference for the mouse (time with mouse vs nonsocial object: WT: $p=0.0082, F_{(1,16)}=9.11$; Src ${ }^{\text {thl/thl }}$ mice: $\left.p=0.0001, F_{(1,14)}=40.42\right)$, but the Src ${ }^{\text {thl/thl }}$ mice spent significantly more time with the mouse com- pared to WT mice (WT $=180.6 \mathrm{~s} \pm 30.89, \mathrm{Src}^{\text {thl/thl }}=$ $\left.310.6 \mathrm{~s} \pm 34.91, p=0.0135, F_{(1,14)}=7.8\right)$ (Fig. $2 A$ ). However, in the test for social novelty preference, only WT mice showed a significant preference for a nonfamiliar mouse (Mouse 2) in comparison with time spent with a familiar mouse (Mouse 1) (time for Mouse 1: Src ${ }^{\text {thl/thl }}=$ $60.90 \mathrm{~s} \pm 13.10$; time for Mouse $2: \mathrm{Src}^{\text {thl/thl }}=153.1 \mathrm{~s} \pm$ 26.30, $\left.p=0.0063, F_{(1,16)}=9.85\right)$ (Fig. 2B). Src ${ }^{\text {thl/thl }}$ mice were not able to distinguish between Mouse 1 and Mouse 2 , suggesting impairment in short term social recognition in Src ${ }^{\text {thl/thl }}$ mice (time for familiar mouse: Src $^{\text {thl/thl }}=192.8$ $s \pm 29.00$, time for nonfamiliar mouse: $S r c^{\text {thl/thl }}=141.0 \mathrm{~s}$ $\left.\pm 27.56, p=0.2165, F_{(1,14)}=1.68\right)$ (Fig. 2B). Number of entries into each compartment was not different between genotypes in each session.

\section{Direct social interaction test}

During a 5 min direct social interaction test, $\mathrm{Src}^{\text {thl/thl }}$ mice spent more time involved in active social approaches than 
WT mice (WT 8.73 $\mathrm{s} \pm 1.66$; Src ${ }^{\text {thl/thl }}: 64.72 \mathrm{~s} \pm 12.21, p$ $\left.=0.0003, F_{(1,16)}=20.66\right)$ (Fig. 2C).

Tube test of social dominance

When WT mice and Src ${ }^{\text {thl/thl }}$ mice were facing each other, $\mathrm{Src}^{\text {thl/thl }}$ mice showed social dominance over approximately $70 \%$ of their matches (WT $=20.00 \pm 9.18 ;$ Src $^{\text {thl/thl }}$ $\left.=80.00 \pm 9.18, p=0.0001, F_{(1,38)}=21.38\right)($ Fig. $2 D)$.

\section{Olfactory test}

Both genotypes showed dishabituation towards a new smell on trial seven of an olfactory test, indicating an intact olfactory system (Fig. 2E). The effect of the genotype was not significant $\left(p=0.3915, F_{(1,102)}=0.77\right)$, but the effect of trial was considered highly significant $(p=$ $\left.0.0001, F_{(1,102)}=15.46\right)$.

\section{Mating induced USVs}

$\mathrm{Src}^{\text {thl/thl }}$ and WT male mice emitted similar numbers of ultrasonic calls during the 5 min encounter with a female mouse. Repeated-measure ANOVA revealed no significant difference between groups across time $(p=0.4512)$ (Fig. 3A).

\section{Social reunion induced USVS}

Most of the female calls during female-female social reunion paradigm occurred in the first $2 \mathrm{~min}$ of the $5 \mathrm{~min}$ trial. A repeated-measure ANOVA over the 5 min revealed a significant difference in number of USV calls between groups $\left(p=0.0044, F_{(1,72)}=10.6\right)$. The interaction between time and genotype was highly significant $(p=$ $\left.0.0001, F_{(4,72)}=9.08\right)$. Src ${ }^{\text {thl/thl }}$ females called more in both the first (WT $=9.27 \pm 3.55$; Src ${ }^{\text {thl/thl }}=160.9 \pm$ $\left.41.04, p=0.001, F_{(1,19)}=14.96\right)$ and second minute (WT $=16.64 \pm 10.79 ;$ Src $^{\text {thl/thl }}=78.00 \pm 23.33, p=0.0235$, $\left.F_{(1,19)}=6.06\right)$ when compared to WT females (Fig. 3B).

$A$ difference was observed in the number of both flat (Type 1) and frequency-modulated (Type 3) USVs between WT and Src ${ }^{\text {thl/thl }}$ female mice (types of calls were described previously by Wang et al, 2008). A repeatedmeasures ANOVA over the $5 \mathrm{~min}$ of the number of Type 1 USV calls revealed a significant difference in the number of calls between groups $\left(p=0.0219, F_{(1,72)}=6.29\right)$ and the interaction between time and genotype was highly significant $\left(p=0.0009, F_{(4,72)}=5.29\right)$. Type 1 calls were significantly higher in $\mathrm{Src}^{\text {thlithl }}$ mice during the first minute of recording only (WT $=4.55 \pm 2.06$; Src ${ }^{\text {thl/thl }}=37.20 \pm$ 11.99, $\left.p=0.0111, F_{(1,19)}=7.91\right)$ (Fig. 3C). No significant effect of genotype $\left(p=0.4164, F_{(1,72)}=0.69\right)$ or time $(p=$ $\left.0.3252, F_{(4,72)}=1.18\right)$ was observed between groups for Type 2 calls (Fig. 3D). However, Type 3 USV calls showed a significant genotype effect between groups $(p=0.0084$, $\left.F_{(1,72)}=8.75\right)$ and the interaction between time and genotype was highly significant $\left(p=0.0002, F_{(4,72)}=6.29\right.$ ). The number of calls were significantly higher in Src ${ }^{\text {thl/thl }}$ female mice during the first $\left(\mathrm{WT}=3.27 \pm 1.14 ; \mathrm{Src}^{\text {thl/thl }}=\right.$ $\left.109.6 \pm 37.53, p=0.0077, F_{(119)}=8.86\right)$ and second minute $\left(\mathrm{WT}=4.64 \pm 2.44 ; \mathrm{Src}^{\text {thlthl }}=51.30 \pm 15.76, p=\right.$ 0.0063, $\left.F_{(1,19)}=9.42\right)$ (Fig. 3E).

\section{Exploratory activity and anxiety}

In the open-field test, there was a significant effect of genotype $\left(p=0.0381, F_{(1,170)}=4.66\right)$ and time interval $(p$ $\left.=0.0006, F_{(5,170)}=4.54\right)$ on the total distance travelled (Fig. 4A), with the $S r c^{\text {thl/thl }}$ mice having a greater total distance travelled and both genotypes travelling more during the first $15 \mathrm{~min}$. Src ${ }^{\text {thl/thl }}$ mice travelled less distance in the center of the arena compared with WT mice $\left(\mathrm{WT}=31.19 \pm 6.76 \% ; \mathrm{Src}^{\text {thl/thl }}=12.89 \pm 3.16 \%, p=\right.$ $0.011, F_{(1,24)}=7.59$, one-way ANOVA) (Fig. $4 B$ ), suggesting an increase in anxiety-like behavior in the $\mathrm{Src}^{\text {thl/thl }}$ mice. However, we did not observe any difference between genotypes in the elevated plus maze, another test for anxiety. The percentage time spent in the open arm was similar in both groups (WT $=4.11 \pm 1.17 \%$; $\mathrm{Src}^{\text {thl/thl }}$ $=4.64 \pm 1.35 \%, p=0.7775, F_{(1,29)}=0.08$, one-way ANOVA) (Fig. 4C). The total distance travelled in the elevated plus maze was not different between the two genotypes.

\section{Sensory and motor function}

We assessed motor coordination, strength, and motor skill learning in Src ${ }^{\text {thl/thl }}$ mice using the balance beam, grip strength, and rotarod tasks. Src ${ }^{\text {thl/thl }}$ mice took significantly longer time to cross the beam (WT $=5.54 \pm 0.84$ $\left.\mathrm{s} ; \mathrm{Src}^{\text {thl/thl }}=11.50 \pm 1.39 \mathrm{~s}, p=0.0011, F_{(1,23)}=13.93\right)$ and had increased number of foot slips while crossing the beam (WT $=0.50 \pm 0.23$; Src ${ }^{\text {thl/thl }}=1.92 \pm 0.31, p=$ $\left.0.0014, F_{(1,23)}=13.30\right)$ (Fig. $\left.5 A, B\right)$ (one-way ANOVA).

We also tested grip strength in the same mice to determine if differences in muscle strength might account for the differences in the balance beam results. This test revealed a significant decrease of grip strength for both forelimb (WT $=98.97 \pm 4.37 \mathrm{~g}$; Src ${ }^{\text {thl/thl }}=80.69 \pm 5.20 \mathrm{~g}$, $\left.p=0.0019, F_{(1,26)}=7.32\right)$ and for forelimb and hindlimb combined $\left(\mathrm{WT} \stackrel{\text {, }}{=} 162.7 \pm 8.62 \mathrm{~g} ; \mathrm{Src}^{\text {thl/thl }}=122.5 \pm 9.60\right.$ $\left.\left.g, p=0.0047, F_{(1,26}\right)=9.56\right)$ in Src ${ }^{\text {thl/thl }}$ mice (Fig. 5C,D) (one-way ANOVA).

We found an overall significant difference in the amount of time mice spent walking on the rotating rod on days 3 and 4 (Day 3: WT $=177.9 \pm 12.52 \mathrm{~s} ;$ Src $^{\text {thl/thl }}=137.1 \pm$ $10.02 \mathrm{~s}, p=0.019, F_{(1,22)}=0.019 ;$ Day $4: \mathrm{WT}=182.7 \pm$ $14.50 \mathrm{~s} ; \mathrm{Src}^{\text {thl/thl }}=124.7 \pm 12.78 \mathrm{~s}, p=0.0065, F_{(1,22)}=$ 9.02) but not on days 1 or 2 (Day 1: WT $=98.42 \pm 11.97$ $\mathrm{s} ; \mathrm{Src}^{\text {thl/thl }}=89.58 \pm 8.70 \mathrm{~s}, p=0.5566, F_{(1,22)}=0.09$; Day 2: WT $=131.6 \pm 12.26 \mathrm{~s} ; \mathrm{Src}^{\text {thl/thl }}=126.2 \pm 12.66 \mathrm{~s}$, $p=0.7615, F_{(1,22)}=0.36$ ) (one-way ANOVA). This suggests a role for Src in rotarod learning rather than rotarod performance (Fig. 5E).

\section{Acoustic startle response}

$\mathrm{Src}^{\text {thl/thl }}$ mice showed a significant enhancement of startle response at the $115 \mathrm{~dB}\left(\mathrm{WT}=242.0 \pm 41.36 \mathrm{AU} ; \mathrm{Src}^{\text {thl/thl }}\right.$ $\left.=471.1 \pm 83.82 \mathrm{AU}, p=0.028, F_{(1,14)}=6.01\right)$ and $110 \mathrm{~dB}$ $\left(\mathrm{WT}=293.2 \pm 13.69 \mathrm{AU} ; \mathrm{Src}^{\text {thl/thl }}=410.3 \pm 36.92 \mathrm{AU}, p\right.$ $\left.=0.01, F_{(1,14)}=8.85\right)$ levels of startling stimulus. However, at the 70-105 dB levels, Src ${ }^{\text {thl/thl }}$ mice exhibited a similar startle magnitude when compared with WT mice ( $p$ $=0.05$ ) (one-way ANOVA) (Fig. 6).

\section{Learning, memory, and attention deficits}

\section{Morris water maze}

Visuospatial learning and memory was assessed using the Morris water maze task. Src ${ }^{\text {thl/thl }}$ mice did not show 
A

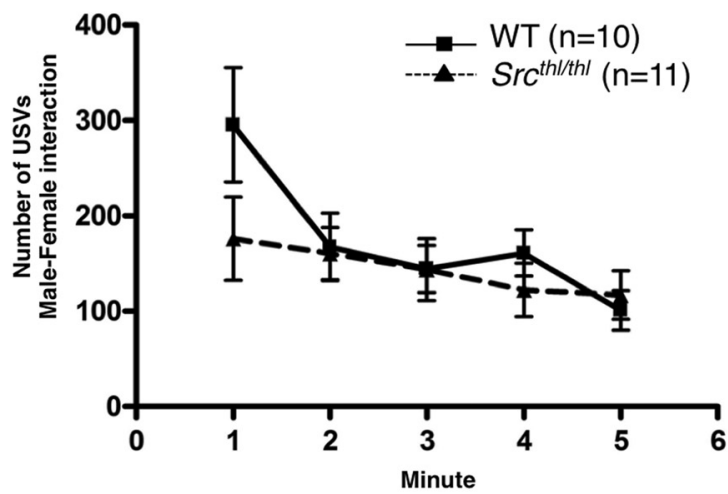

C

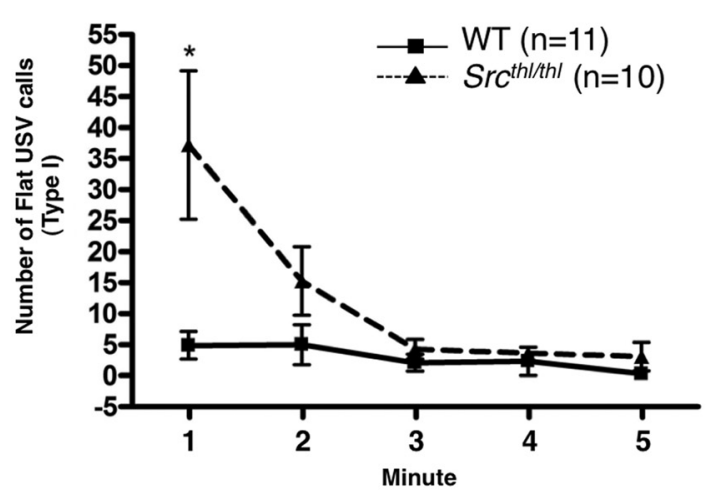

E

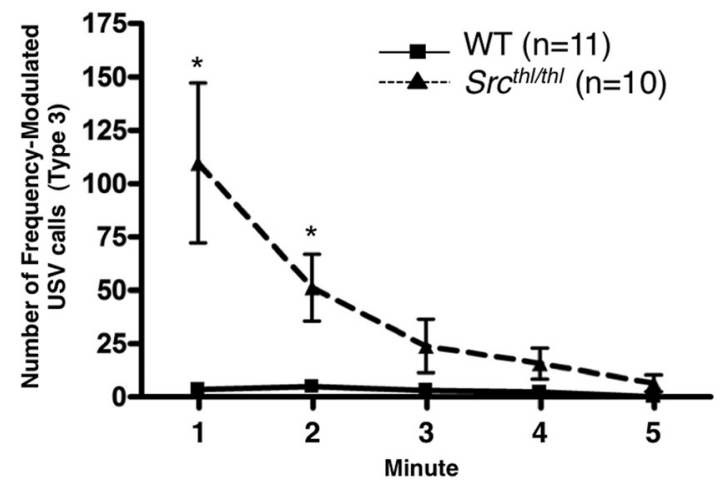

B

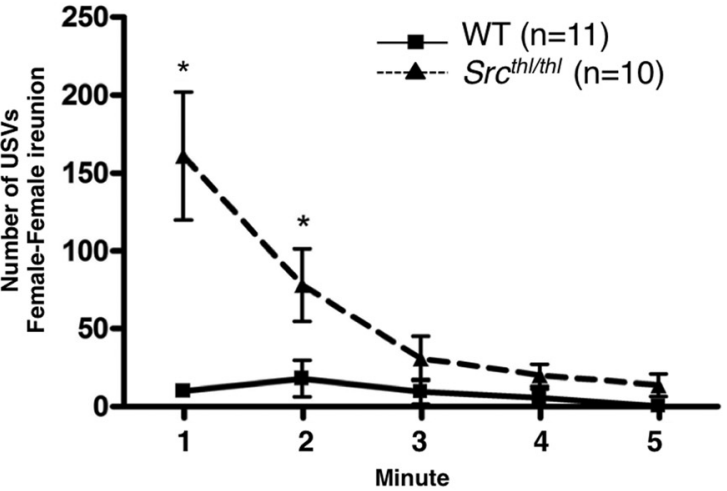

D

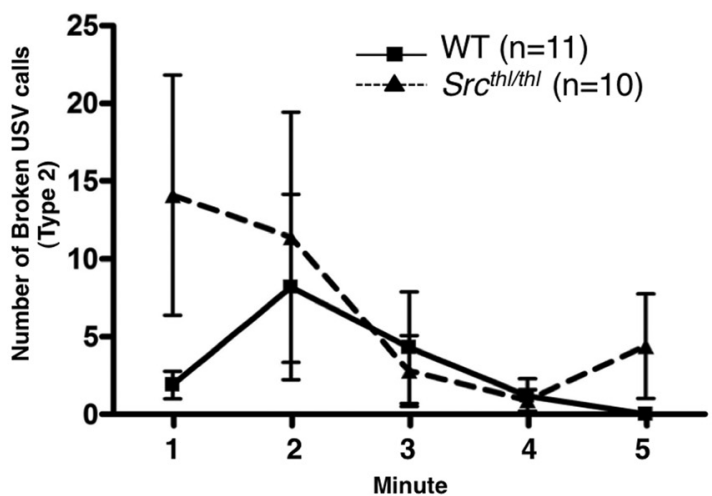

Figure 3. Enhanced social reunion of female Src ${ }^{\text {thl/thl }}$ mutant mice. A, Time course of total number of USVs in WT and Src ${ }^{\text {thl/thl }}$ male mice when introduced to females for a $5 \mathrm{~min}$ trial. A gradual decline in total number of calls was observed across time but the total number of calls was still high, with no significant difference between WT and Srcth/thl mice. Data are presented as mean \pm SEM for each time point $\left(n=10 \mathrm{WT}, n=11 \mathrm{Src}^{\text {thl/thh}}\right)$. B. Time course of total number of USVs in WT and Src ${ }^{\text {thlthl }}$ females when reunited with a litter mate for a 5 min trial. A gradual decline in total number of calls is observed across time with a significant difference between WT and Srcthltthl females in total number of USVs produced in the first and second minutes $\left(n=11 \mathrm{WT}, n=10 \mathrm{Src} c^{\text {thl/thl }}\right)$. C. There is a significant difference in the number of flat (Type 1) USVs produced by WT versus Src ${ }^{\text {thl/thl }}$ females at minute $1(n=11$ WT, $n=$ $\left.10 \mathrm{Src}^{\text {thl/thl}}\right)$. $\boldsymbol{D}$. There was no significant difference in the number of broken (Type 2) USVs produced by WT versus Src ${ }^{\text {thl/thl }}$ females across time $\left(n=11 \mathrm{WT}, n=10 \mathrm{Src}{ }^{\text {thl/thh}}\right)$. $\boldsymbol{E}$. There is a significant difference in the number of frequency modulated (Type 3 ) USVs produced by WT versus Src ${ }^{\text {thl/thl }}$ females at minutes 1 and $2(n=11 \mathrm{WT}, n=10 \mathrm{Src}$ th/thh $)$. Data are presented as mean \pm SEM for each time point. $* p<0.05$ between genotypes.

impaired motor performance or decreased motivation to escape, as all mutants showed similar escape latencies in the visible and the hidden platform task $p=0.4648$, two-way ANOVA) (Fig. 7A). There was a significant effect of genotype ( $\left.p=0.001, F_{(1,22)}=22.32\right)$ but no effect of target $\left(p=0.3926, F_{(1,22)}=0.76\right)$ (two-way ANOVA) in the probe trial given after $6 \mathrm{~d}$ of training. WT mice showed significantly more crossings of the target platform position 

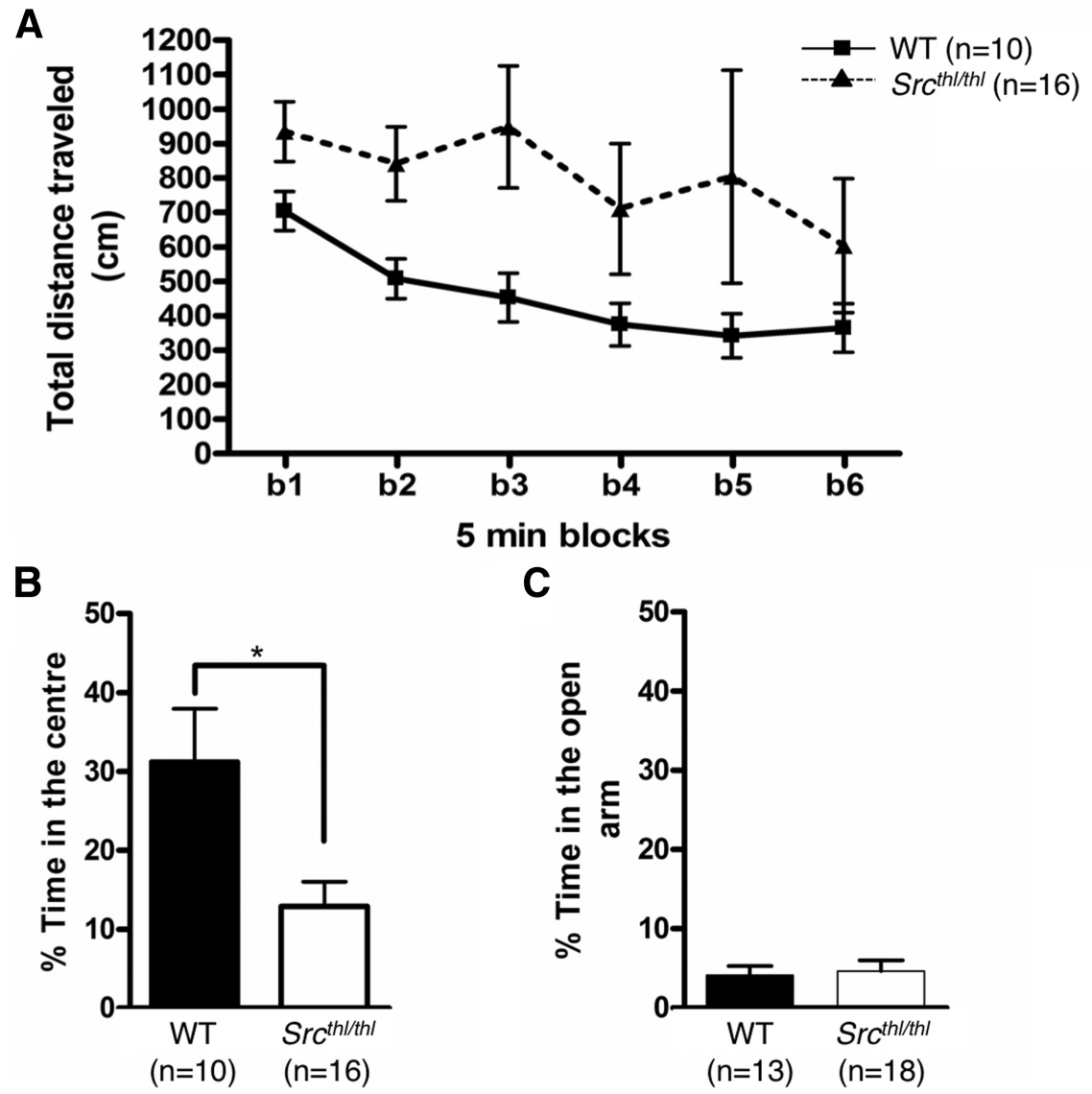

Figure 4. Src ${ }^{\text {thl/thl }}$ mutant mice show hyperactivity in the open field. $\boldsymbol{A}, \operatorname{Src}^{\text {thl/thl }}$ mutant mice showed an increase in total distance travelled in the open field compared with WT mice ( $n=10$ WT (5 males, 5 females), $n=16 \mathrm{Src}^{\text {thl/thl }}(9 \mathrm{males}, 7 \mathrm{females})$ ). $\boldsymbol{B}$, Percentage of time spent in the center of the arena versus the entire open field was decreased in Src ${ }^{\text {thl/thl }}$ ( $n=10$ WT ( 5 males, 5 females), $n=16 \mathrm{Src}^{\text {thl/thl }}$ (9 males, 7 females)). $* p<0.05$ between genotypes. $\boldsymbol{C}$, Percentage time spent in the open arms versus the closed arms of the elevated plus maze showed no significant differences between genotypes $(n=13$ WT ( 7 males, 6 females), $n=$ $18 \mathrm{Src}^{\text {thl/thl }}$ (9 males, 9 females)). Data are presented as mean \pm SEM.

compared with similarly sized and positioned areas in the other quadrants (crossing in target platform quadrant: $4.42 \pm 0.514$, averaged crossing in nontarget quadrants: $\left.2.72 \pm 0.27, p=0.0082, F_{(1,22)}=8.44\right)$, whereas Src ${ }^{\text {thl/thl }}$ mice did not show any preference for the target location (crossing in target platform quadrant: $1.42 \pm 0.42$, averaged crossing in nontarget quadrants: $2.33 \pm 0.38, p=$ $0.1167, F_{(1,22)}=2.67$ ) (Fig. $7 B$ ).

\section{Fear conditioning}

Levels of freezing in response to both a context- and cued-fear CS was measured. During the context test, $S \mathrm{Sc}^{\text {thl/thl }}$ mice displayed similar periods of freezing relative to WT mice. In the CS test, Src ${ }^{\text {thl/thl }}$ mice displayed shorter freezing time relative to WT. These findings suggest that $\mathrm{Src}^{\text {thl/thl }}$ mice have impaired learning and/or memory performance. Cued fear conditioning is the most widely used behavioral task to assess amygdala function (Phelps and LeDoux, 2005). Src ${ }^{\text {thl/thl }}$ mice showed impaired fearrelated learning during cued fear conditioning. Mice were conditioned to two pairs of a tone (CS) and a footshock (US) on the training day. Prior to pairing, there was a moderate difference between groups in baseline freezing
$\left(\mathrm{WT}=3.14 \pm 0.73 \% ; \mathrm{Src}^{\text {thl/thl }}=7.87 \pm 1.32 \%, p=\right.$ $0.0026, F_{(1,44)}=10.21$, one-way ANOVA) and freezing in response to unpaired tone (WT $=5.79 \pm 1.69 \%$; Src ${ }^{\text {thl/thl }}$ $=13.97 \pm 2.74 \%, p<0.05, F_{(1,44)}=6.70$, one-way ANOVA). However, the post-shock freezing was similar between groups $\left(\mathrm{WT}=29.21 \pm 3.93 \%\right.$; Src thl/thl $^{\text {L }}=28.98$ $\pm 3.24 \%, p=0.9633, F_{(1,44)}=0.04$ ) (Fig. $4 C$ ), suggesting that the Src mutation alters general anxiety to some extent. At $24 \mathrm{~h}$ after pairing, the mice were tested for longterm contextual and cued fear memory. Subjects were placed in a novel chamber for 3 min prior to the presentation of the tone; both groups of mice displayed only weak freezing in the novel chamber (WT $=5.79 \pm 1.69 \%$; $S r c^{\text {thl/thl }}=13.97 \pm 2.74 \%, p \leq 0.0130, F_{(1,44)}=6.70$, one-way ANOVA) (Fig. 7C). During the tone delivery, $\mathrm{Src}^{\text {thl/thl }}$ mice demonstrated weaker freezing (38.48 \pm $4.26 \%)$ in comparison to WT mice $(57.68 \pm 3.98 \%, p=$ $\left.0.0019, F_{(1,47)}=10.88\right)$ (Fig. $\left.7 D\right)$, suggesting that Src function is important for long-term memory of the CS-US association. In contrast, contextual fear was not effected in Src ${ }^{\text {thl/thl }}$ mice (WT $=50.14 \pm 5.65 \% ;$ Src $^{\text {thl/thl }}=42.08 \pm$ $4.50 \%, p=0.2728, F_{(1,47)}=1.23$, one-way ANOVA). 
A

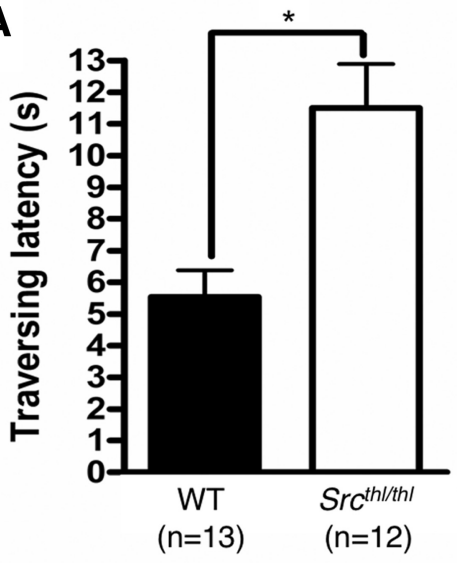

B

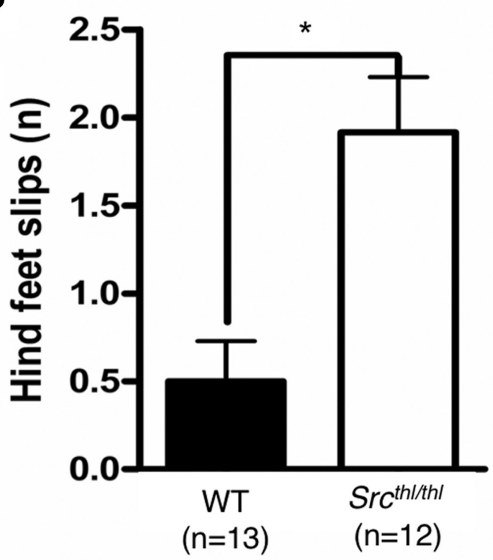

C

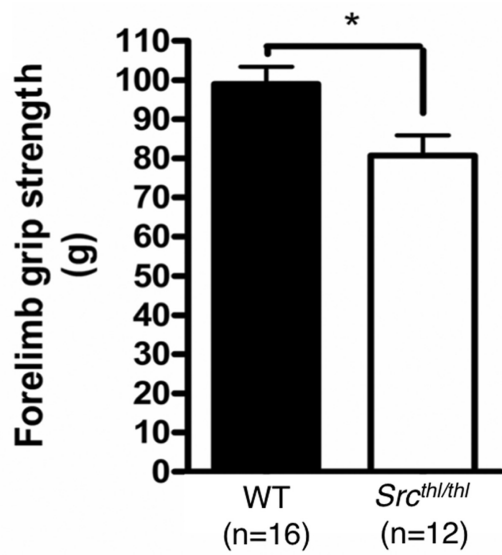

D

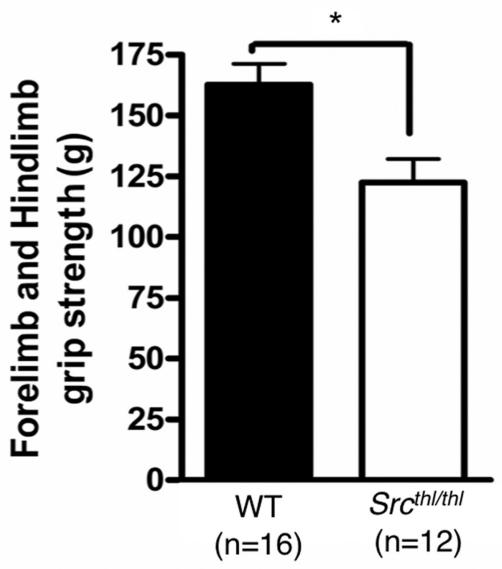

\section{E}

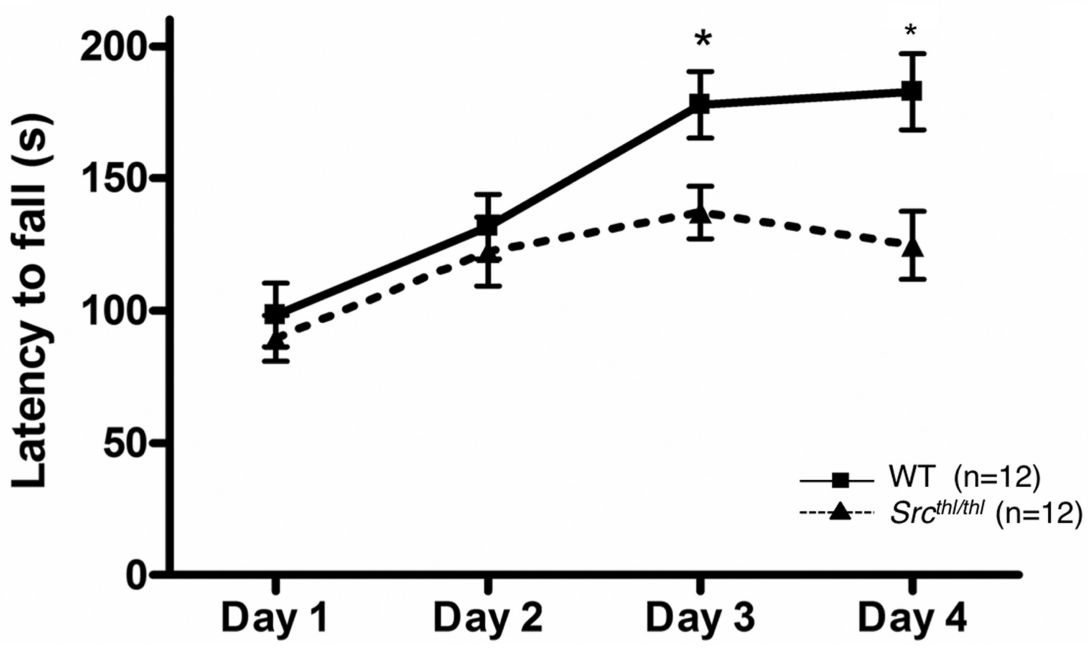

Figure 5. Balance and motor strength deficiency in $S r c^{\text {thl/thl }}$ mice and the inability to learn motor skills. Quantitative analysis of raised balance beam, grip strength, and rotarod task in WT and Src ${ }^{\text {thl/thl }}$ mice. $\boldsymbol{A}, \mathrm{Src}^{\text {thl/thl }}$ mice needed significantly more time to cross the balance beam than did WT mice ( $n=13$ WT (7 males, 6 females), $n=12$ Src $^{\text {thl/thl }}$ ( 6 males, 6 females)). $\boldsymbol{B}$, Src ${ }^{\text {thl/thl }}$ mice had significantly more hindfoot slips than did WT mice. C, Grip strength analysis revealed significantly reduced forelimb grip strength in Src ${ }^{\text {thl/thl }}$ mice compared with WT animals $(n=16$ WT (9 males, 7 females), $n=12 \mathrm{Src}^{\text {thl/thl }}$ (6 males, 6 females)). $\boldsymbol{D}$, Grip strength analysis revealed significantly reduced combined forelimb and hindlimb grip strength in Src ${ }^{\text {thl/thl }}$ mice compared with WT animals ( $n=16$ WT ( 9 males, 7 females), $n=12$ Src ${ }^{\text {thl/thl }}$ ( 6 males, 6 females)). E, Mice were tested on an accelerating rotarod with the same speed for 4 consecutive days. The latencies from rotation onset until the mice fell off the rod were measured. WT mice managed to stay significantly longer on the accelerating rotarod than Srcthlthl mice on days 3 and 4 . The falling latencies were similar in both groups on days 1 and 2 . Falling latencies were compared within genotype between days 1 and 4 ( $n=12$ WT ( 6 males, 6 females), $n=12 \mathrm{Src}^{\text {thl/thl }}$ (6 males, 6 females)). Data are presented as mean \pm SEM. $* p<0.05$. 


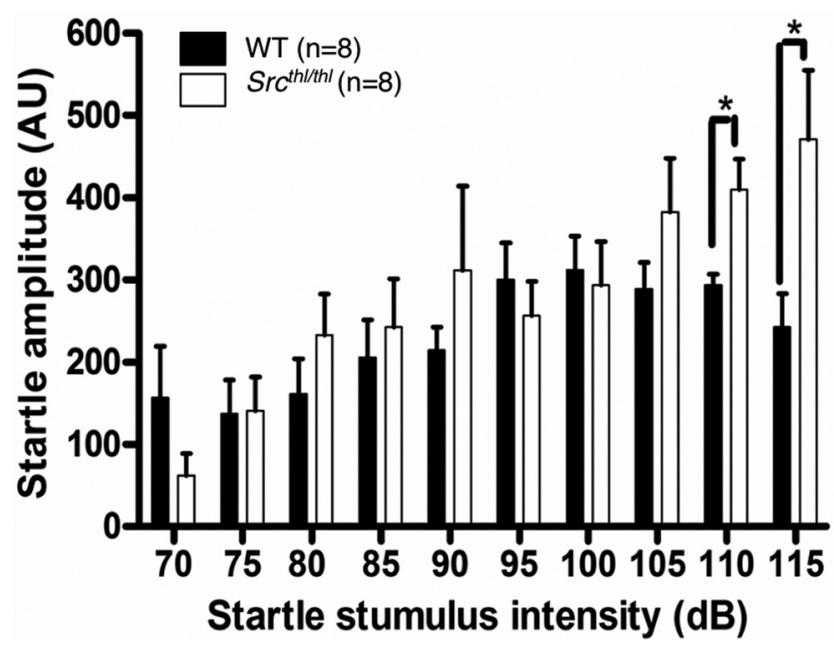

Figure 6. Acoustic startle in response to high startling stimulus is increased in Src ${ }^{\text {thl/thl }}$ mice. Both the WT and Srchl/thl mice exhibited similar startle amplitude responses to the low-intensity acoustic stimuli (70-105 dB). Src ${ }^{\text {thl/thl }}$ mice showed higher responses to the high-intensity stimuli $(110-115 \mathrm{~dB})(n=8$ WT $(8$ males), $n=8 \operatorname{Src}^{\text {thl/thl }}$ (18 males)). $* p<0.05$.

\section{Shock threshold}

Because pain sensitivity can also affect the strength of associative learning, we measured pain threshold [data not shown, $n=10$ WT (5 males, 5 females), $n=10$ $\mathrm{Src}^{\text {thl/thl }}$ (5 males, 5 females)]. The minimum footshock intensity required to produce an audible vocalization response was unaltered in Src ${ }^{\text {thl/thl }}$ mice. (WT $=0.29 \pm 0.01$ $\mathrm{s} ; \mathrm{Src}^{\text {thl/thl }}=0.29 \pm 0.01 \mathrm{~s}, p=0.5560, F_{(1,18)}=0.36$, one-way ANOVA), suggesting that they have similar sensitivity to painful stimuli.

\section{Visual object and novelty recognition \\ The exploration time of displaced objects and novel ob- jects was evaluated. Src ${ }^{\text {thlthl }}$ mice demonstrated an in- ability to selectively react to a spatial and novelty change in the environment. There was a main effect of genotype, with an increase in exploratory preference for spending more time with the displaced object $(>50 \%)$ than the nondisplaced objects displayed by WT mice, whereas $\mathrm{Src}^{\text {thl/thl }}$ mice explored both object categories for a similar amount of time ( 50\%) (WT = 81.08 $\pm 2.61 \%$; Src ${ }^{\text {thl/thl }}=$ $58.11 \pm 3.20 \%, p=0.0001, F_{(1,25)}=28.81$, one-way ANOVA) (Fig. 8A). In the novel object session, when one of the objects was substituted, $S r c^{\text {thl/thl }}$ mice demonstrated an inability to selectively react to a novelty change. There was a main effect of genotype (WT $=87.06 \pm 1.45 \%$; $\mathrm{Src}^{\text {thl/thl }}=58.31 \pm 5.02 \%, p=0.0001, F_{(1,24)}=26.39$, one-way ANOVA) (Fig. 8B).}

\section{Cellular localization of TFII-I and TRPC3}

\section{Western blot analysis}

We performed Western blot analysis of fractionated cellular compartments to compare the distribution of TFII-I and TRPC3 within the cell. Since TFII-I has been shown to require phosphorylation by $\mathrm{Src}$ to translocate to the nucleus in transformed cell lines (Cheriyath et al,
2002), we quantified TFII-I within the nuclear fraction of whole brain from WT and Srchl/thl mice. There was significantly less TFII-I localized to the nucleus in cells from the Src ${ }^{\text {thl/thl }}$ mice compared with WT mice (quantification relative to loading control, WT $=1.03 \pm 0.26$; $S r c^{\text {thl/thl }}=0.44 \pm 0.11$, Student's $t$ test $p<0.05$; Fig. $9 A, C)$. TRPC3 is cycled to the plasma membrane through a process that is regulated by phosphorylated TFII-I within the cytoplasm (Caraveo et al, 2006). We found significantly more TRPC3 contained within the membrane fraction in cells from the Srcthl/thl mice compared with WT mice (quantification relative to loading control, WT $=0.69 \pm 0.14 ; \mathrm{Src}^{\text {thl/thl }}=1.44 \pm 0.16$, Student's $t$ test $p<0.05$; Fig. 9B,C).

\section{Immunocytochemistry}

To support our findings of altered TFII-I and TRPC3 cellular localization by Western blot analysis, we performed immunocytochemistry and confocal imaging on primary cortical neurons from Src ${ }^{\text {thl/thl }}$ mice and their WT littermates, and compared the expression of TRPC 3 and TFII-I in the membrane, cytosol, and nucleus (Fig. 10A,B). We found that TFII-I was significantly less abundant in the nucleus (Fig. 10E, $p=0.00007, F=1.42$, one-way ANOVA) in Src ${ }^{\text {thl/thl }}$ mice but showed higher expression in the cell membrane (Fig. 10C, $p=0.001, F=11.98$, one-way ANOVA). TFII-I levels in the cytosol were not significantly different between $\mathrm{Src}^{\text {thl/thl }}$ mice and their WT littermates (Fig. $10 D, p=0.237, F=1.42$, one-way ANOVA). WT mice showed a broad expression of TFII-I in the nucleus and cytosol, as evidenced by only a $17 \%$ difference in fluorescence between the nucleus and cytoplasm, whereas $\mathrm{Src}^{\text {thl/thl }}$ mice showed a $30 \%$ difference in fluorescence (Fig. 10F, $p=0.014, F=6.4$, one-way ANOVA).

There was also a significant difference in TRPC3 expression between $S r c^{\text {thl/thl}}$ and WT mice. Src ${ }^{\text {thl/thl }}$ mice had an increased level of TRPC3 in both membrane and cytosol (Fig. 10G: $p=0.00001, F=22$; Fig. 10H: $p=$ $0.0000007, F=31$, one-way ANOVA), in contrast with the low expression of TRPC3 in the nucleus (Fig. 10/, $p=$ $0.0000000000003, F=79$, one-way ANOVA). WT mice showed only a $17 \%$ difference in fluorescence between the nucleus and cytoplasm, whereas $\mathrm{Src}^{\text {thl/thl }}$ mice showed a $36 \%$ difference in fluorescence (Fig. 10J, $p=$ $0.00000000001, F=74$, one-way ANOVA).

\section{Discussion}

Disruption of both upstream and downstream components of the Src signaling pathway have been linked with human genetic disorders whose behavioral phenotypes include alterations in social interaction and cognition. We hypothesized that altered Src function could contribute to phenotypes that overlap with those found in WBS or autism spectrum disorder (ASD), and that these phenotypes might, at least in part, be due to altered TFII-I phosphorylation by Src. Here we report that mice lacking Src exhibit a complex behavioral and cognitive phenotype that does indeed exhibit overlap with WBS.

The Src mutant mice show craniofacial abnormalities and growth retardation, both apparent in WBS. The mice 
A

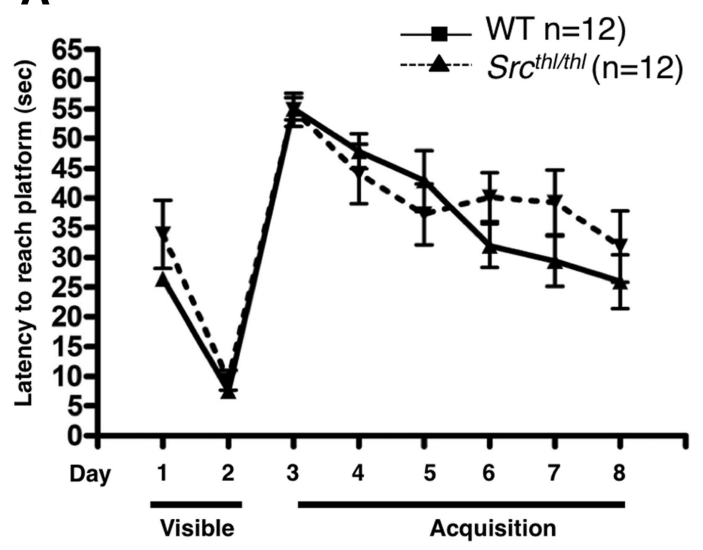

C

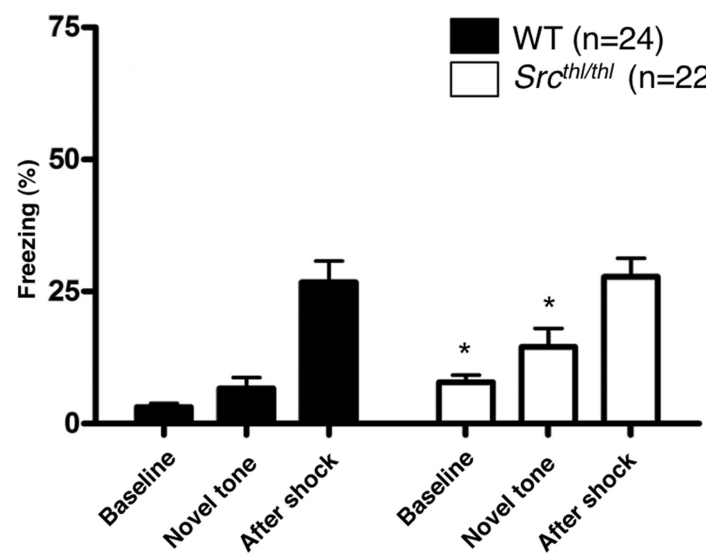

B

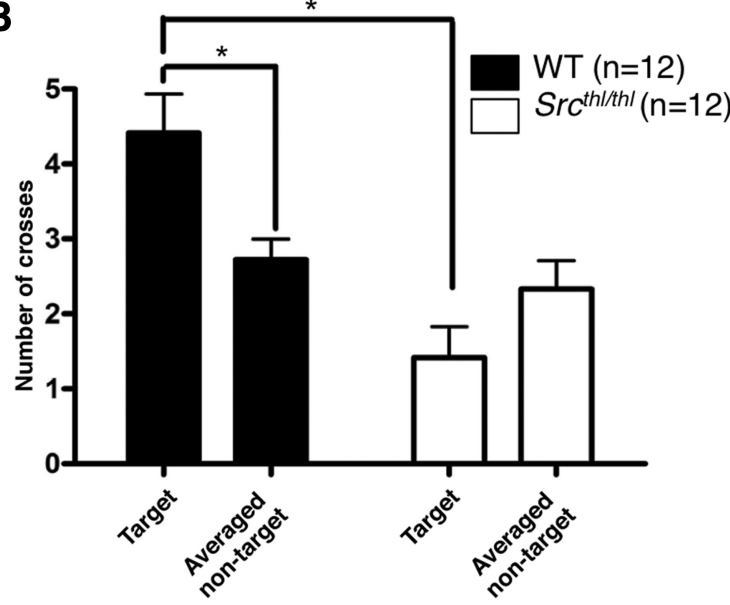

D

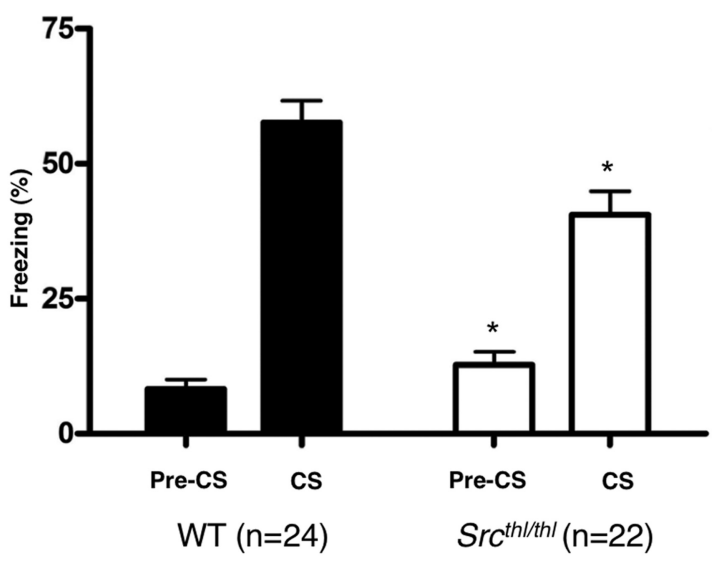

Figure 7. Impaired memory in Src ${ }^{\text {thl/thl }}$ mutant mice. A, Mice were assessed in the Morris water maze procedure $(n=12$ WT (12 males), $n=12 \mathrm{Src}^{\text {thl/thl }}$ (12 males)). Mean latency to reach a target platform in a visible platform session (days 1 and 2$)$, and in a hidden-platform acquisition phase (days 3-8). $\boldsymbol{B}$, Spatial memory retention was assessed in the probe trials administered $2 \mathrm{~h}$ after day 8 ( $n=12$ WT (12 males), $n=12$ Src $^{\text {thl/thl }}(12$ males)). Mean number of platform crosses during probe trial 1 are shown. $* p<0.05$. $\boldsymbol{C}$, Freezing (as a percentage of total time) in a novel conditioning chamber ( $n=24$ WT (13 males, 11 females), $n=22$ Src ${ }^{\text {thl/thl }}$ (12 males, 10 females)). After recording baseline freezing for $120 \mathrm{~s}$, a tone was presented for $30 \mathrm{~s}$ (novel tone), after which mice were given a footshock and left inside the chamber for another $30 \mathrm{~s}$ (after shock). A subtle increase in freezing was observed during baseline and novel tone presentation in Src ${ }^{\text {thl/thl }}$ mice. $* p<0.05$. $D$, Cued fear memory $24 \mathrm{~h}$ after pairing $(n=24$ WT $(13$ males, 11 females), $n=$ $22 \mathrm{Src}^{\text {thl/thl }}$ (12 males, 10 females)). Pre-CS is freezing in the absence of the tone in a novel context, while CS is percentage freezing during tone presentation. Src ${ }^{\text {thl/thl }}$ mice showed impaired auditory fear conditioning, however, context was not affected. Data are presented as mean \pm SEM, $* p<0.05$.

have a short cranial base and defects in incisor and molar tooth formation and eruption. Individuals with WBS have a shortened posterior cranial base and thickened frontal and occipital bones (Axelsson et al, 2005), reminiscent of the thickened calvaria and osteopetrosis observed in Src mutants (Soriano et al, 1991). Tooth development is also aberrant in WBS. Tooth shape is tapered and the teeth are usually more widely spaced, with $\sim 40 \%$ of individuals having agenesis of at least one permanent tooth, and $12 \%$ having at least six missing teeth (Axelsson, 2005). Mice with hemizygous deletion of Gtf2i do not show an overt craniofacial abnormality or tooth defects (Lucena et al, 2010; Sakurai et al, 2011), however, it is possible that altered TFII-I phosphorylation due to lack of Src has a larger impact than Gtf2i hemizygosity. Unfortunately, Gtf2i homozygous null embryos die well before tooth-bud for- mation with neural tube defects and exencephaly (Enkhmandakh et al, 2009; Sakurai et al, 2011), making phenotypic assessment of tooth or craniofacial development in animals with lower levels of TFII-I impossible. In mice, all GTF2I family member genes (Gtf2i, Gtf2ird1, and Gtf2ird2) showed strong expression in the developing teeth (Ohazama and Sharpe, 2007), and evidence from both mice and humans suggest that GTF2IRD1 is involved in craniofacial development (Tassabehji et al, 2005; Howard et al, 2012), suggesting that there could be downstream effects in common with Src.

We also observed differences in social behavior in the $\mathrm{Src}^{\text {thl/thl }}$ mice that were similar to those observed in people with WBS. Sociability was increased in four different social affiliation tasks in Src ${ }^{\text {thl/thl }}$ mice (direct social interaction, social approach, tube test, and social reunion), 

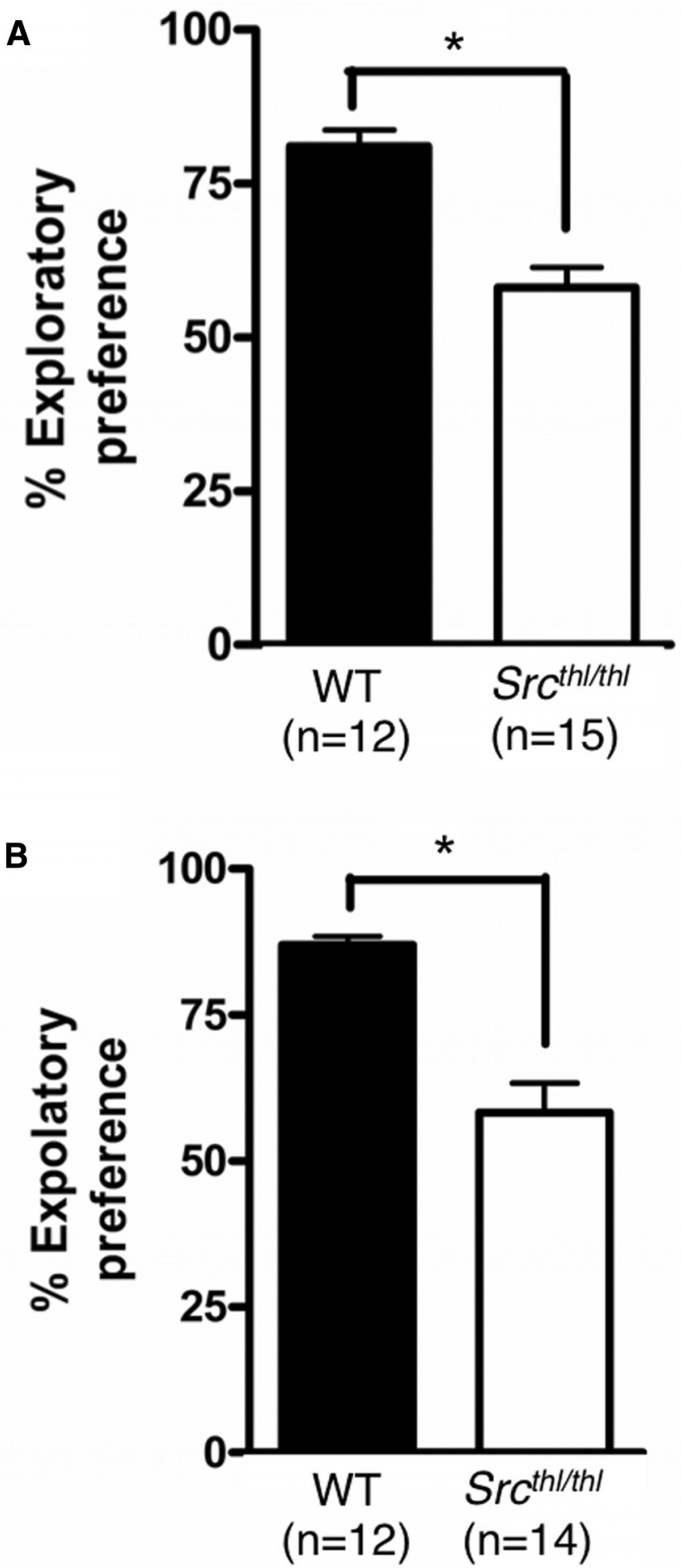

Figure 8. Src ${ }^{\text {thl/thl }}$ mice have a deficit in short term object recognition. A, Mean time (s) spent exploring the displaced versus nondisplaced objects in the spatial change session ( $n=12$ WT (6 males, 6 females), $n=15 \operatorname{Src}^{\text {thl/thl }}$ (8 males, 7 females)). $\boldsymbol{B}$, Mean time spent exploring the novel item versus three familiar objects in the nonspatial change session ( $n=12$ WT ( 6 males, 6 females), $n=14 \mathrm{Src}^{\text {thl/thl }}$ (7 males, 7 females). Data are expressed as mean \pm SEM, $* p<0.05$. suggesting that Src plays a significant role in modulating social interaction. Interestingly, although the Src ${ }^{\text {thl/thl }}$ mice showed an increased social dominance in the tube test, this was unlikely the result of increased aggression, since we often observed the Srcthl/thl mice standing passively in the middle of the tube, looking at the WT mice (personal observation). The WT mice tried to escape by moving under the Src mutant mice, however, and after several unsuccessful attempts they backed out of the tube. This finding is in agreement with a study that found social motivation in children with WBS was not driven by a desire for social dominance, unlike in typically developing children ( $\mathrm{Ng}$ et al, 2014). In the social reunion of female mice paradigm, there was an increase in the number of calls produced by Src ${ }^{\text {thl/thl }}$ females in the first 2 min of the test, compared to WT females, whereas the number of USVs was unchanged in the mating-induced USV experiment, indicating that loss of $\mathrm{Src}$ is related to increased social interest but not reward-seeking behaviors such as courtship and mating (Gourbal et al, 2004; Moles et al, 2007; Lahvis et al, 2011).

The Src mutant mice showed increased social approach, which may be similar to the increased social approach documented in individuals with WBS (Doyle et al, 2004). However, they failed to show a preference for the novel mouse in the social short-term recognition section of this task, unlike individuals with Williams syndrome who have a heightened approachability toward strangers (Doyle et al, 2004). Lack of preference for a novel mouse is usually an indication of impaired social recognition, but in this case it may reflect a general problem with cognitive function, as evidenced by their failure to show a preference for a novel object or an object that has moved positions (Fig. 8). Mice heterozygous for Gtf2i also showed deficits in novel object recognition and social habituation, as well as increased social interaction (Sakurai et al, 2011), similar to the Srcthlthl mice.

The possible link between Src function and social interaction is intriguing. Src itself is tightly regulated by c-Src tyrosine kinase (CSK), which inhibits Src function through phosphorylation of $\mathrm{C}$-terminal tyrosine residues (Okada et al, 1991; Nada et al, 1993). Interestingly, CSK lies within a region of human chromosome 15q24 that is deleted in some individuals with ASD (Marshall et al, 2008; Mclnnes et al, 2010). ASD has also been reported in individuals with duplication of the WBS region (7q11.23 duplication syndrome, Dup7) (Berg et al, 2007; Van der Aa et al, 2009; Levy et al, 2011; Sanders et al, 2011) and a GTF2l intronic haplotype has been associated with ASD (Malenfant et al, 2012). It could be hypothesized, therefore, that upregulation of TFII-I function, either through gene duplication or through disruption of Src inhibition, might contribute to decreased social interaction.

We assessed exploratory activity and anxiety-related behaviors using the open-field test and the elevated plus maze. Src ${ }^{\text {thl/thl }}$ mice showed increased activity, a common finding in WBS (Mervis and John, 2010), but the anxiety assessment was inconclusive: they spent significantly less time in the center of the open field compared to WT littermates, but showed no genotype differences in the 

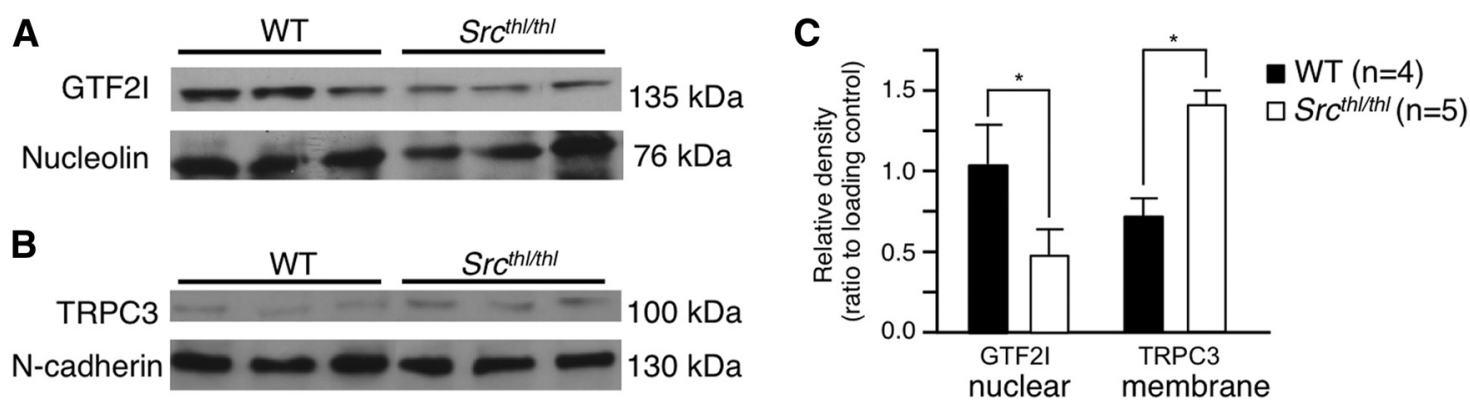

Figure 9. Altered cellular localization of TFII-I and TRPC3 in Src ${ }^{\text {thl/thl }}$ mouse brain tissue. Western blot analysis was carried out on fractionated cell lysates from WT and Src ${ }^{\text {thl/thl }}$ adult mouse whole brain. Representative blots are shown ( $\left.n=4 \mathrm{WT}, n=5 \mathrm{Src} c^{\text {thl/thl}}\right)$. $\boldsymbol{A}$, The nuclear fraction was probed with TFII-I antibody, with nucleolin as a loading control. $\boldsymbol{B}$, The membrane fraction was probed with TRPC3 antibody, with $n$-cadherin as a control. $\boldsymbol{C}$, Quantification of the Western blots. Data are expressed as mean \pm SEM, $* p$ $<0.05$.

plus maze. In the startle response, Src ${ }^{\text {thl/thl }}$ mice exhibited greater reaction to high-intensity stimuli $(110 \mathrm{~dB}$ and 115 $\mathrm{dB}$ ) than WT mice, suggesting either sensitivity to loud noises (decreased acoustic threshold) or a decrease in the sound level necessary to provoke a startle response (decreased response threshold). The elevated startle response could arise from an abnormality in secondary brain regions that modulate the primary startle response, either through intrinsic disturbance such as altered synaptic connections, or indirectly, through an increase in arousal, rather than a change in the primary sensory response of the auditory circuit. The presentation of overresponsiveness to sensory stimuli in individuals with WBS supports a potential role for Src signaling in the modulation of sensory input and the acoustic startle reflex. Studies in individuals with WBS have suggested that the hypersensitivity to sound may be due to hyperexcitability of the auditory efferent system, coupled with diminished or absent acoustic reflexes (Attias et al, 2008).

Intellectual disability is common in WBS, but some cognitive abilities are more affected than others, and in particular deficits in visuospatial constructive cognition are a hallmark of WBS (Mervis, 1999). Impairment in visuospatial learning and memory of Src mutant mice was apparent in the probe trial given after $6 \mathrm{~d}$ of training, where the mice showed a significant decrease in the number of crossings over the learned position of the platform compared to WT mice (Fig. 7B). The smaller size of the mice could make comparison to WT littermates problematic in behavioral paradigms where motor performance is important. The mutant mice had mild deficits in motor performance and learning when assessed on the rotarod and balance beam (Fig. 5), suggesting that they could be experiencing fatigue due to greater muscle weakness. Alternatively, since their rotarod performance was equivalent to WT mice for the first $2 \mathrm{~d}$ of training, the impairment may be in motor learning rather than motor performance. The mutant mice showed similar escape latencies in the visible and hidden platform task of the water maze test, and their swim speed was similar to that of WT mice (Fig. 7A), suggesting that the visuospatial learning deficit was not due to impaired motor performance or decreased motivation to escape. Spatial learn- ing and memory has been linked with hippocampal function in mice (Phelps and LeDoux, 2005), but in WBS, functional imaging has linked visuospatial construction deficits with abnormal processing in the dorsal stream around the intraparietal sulcus (Meyer-Lindenberg et al, 2004).

Amygdala dysfunction has also been demonstrated in WBS using functional imaging, and Src mutant mice exhibited impaired cued fear conditioning, which is dependent primarily upon amygdala-based learning, though contextual fear conditioning was unaffected, suggesting relatively intact hippocampal function (Phelps and LeDoux, 2005; Kim and Jung, 2006). Src is an abundant tyrosine kinase that phosphorylates, and therefore regulates, numerous proteins (Bromann et al, 2004), including NMDA receptors, which it regulates through phosphorylation and subsequent surface expression of NMDA receptor 2 B (NR2B) (Lu et al, 1998; Zhang et al, 2008). It has been previously demonstrated that inhibition of SrC through injection of a peptide that blocks its interaction with NMDA receptors also results in deficits in amygdaladependent learning and long-term potentiation (Sinai et al, 2010), suggesting that in the Src mutant mice, this deficit is due to reduced surface expression of NR2B.

Src has been shown to phosphorylate TFII-I on tyrosines 248 and 611, thereby both translocating it to the nucleus and making it transcriptionally active (Cheriyath et al, 2002). Since the Src mutant mice showed overlap in phenotype with individuals who have WBS, we investigated TFII-I subcellular localization and found a $>50 \%$ increase in TFII-I localized to the plasma membrane, as well as a decrease of $\sim 40 \%$ from WT levels in the nucleus of neurons from Src ${ }^{\text {thl/thl }}$ mice (Figs. 9, 10). These findings are in direct contrast to previous in vitro data that showed increased nuclear localization of TFII-I upon inactivation of Src (Cheriyath et al, 2002). However, our experiments were carried out in primary neuronal cells rather than transformed fibroblast cell lines, and there are likely to be differences in Src function and TFII-I regulation between different cell types, and between primary cells and transformed lines. Our findings suggest that lack of phosphorylation by Src has a direct effect on the cellular 
A
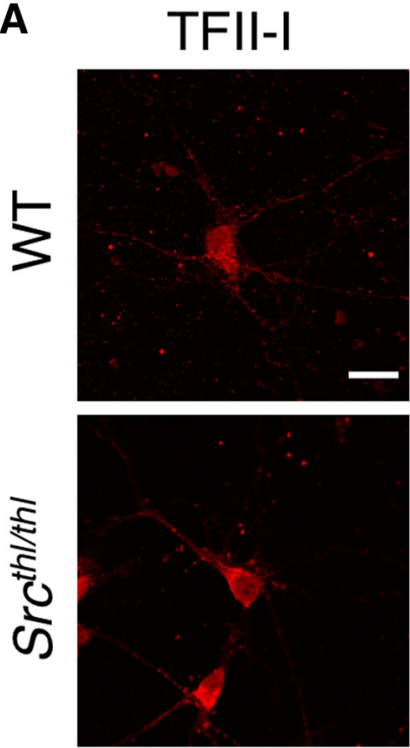

B

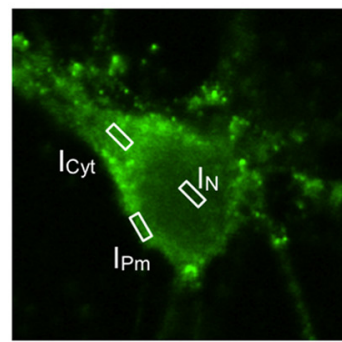

C

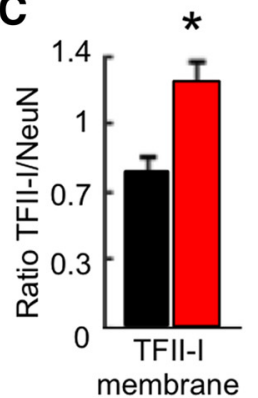

G

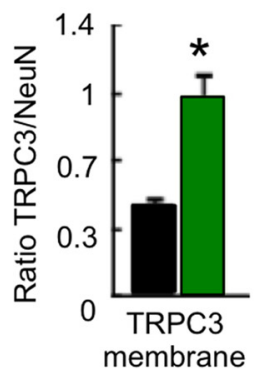

D

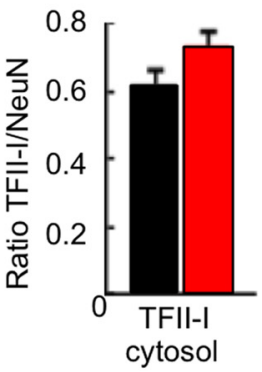

H

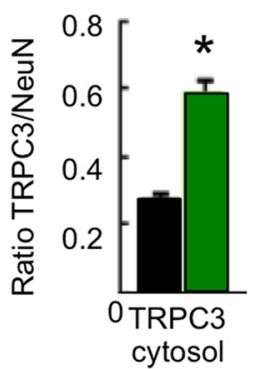

E

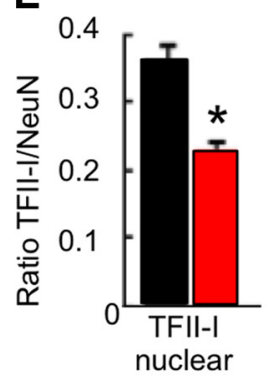

I

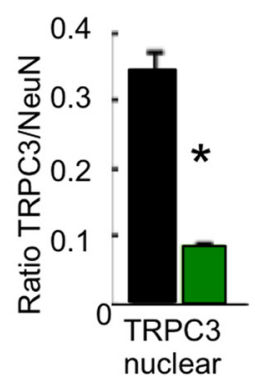

F

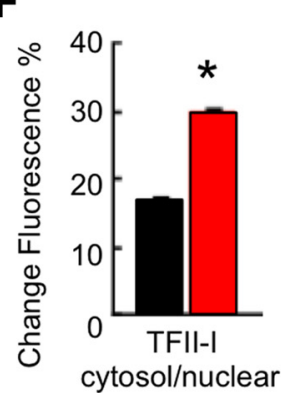

J

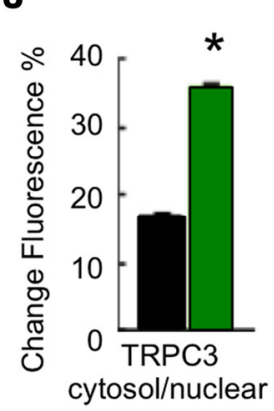

Merge
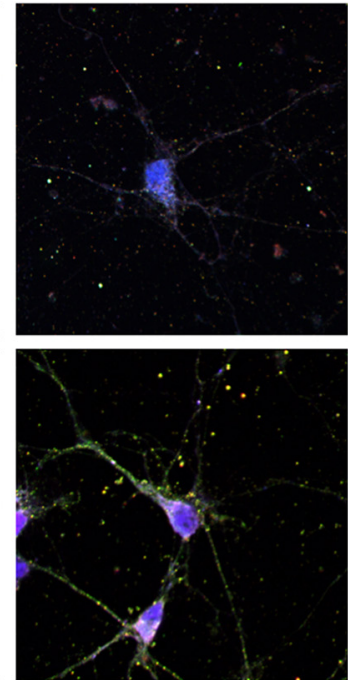

Figure 10. Altered cellular localization of TRPC3 and TFII-I in cultured cortical neurons. TFII-I and TRPC3 fluorescence intensity was calculated from pooled data derived from two independent experiments, each using 10 pups from two different litters $(n=60)$. $\boldsymbol{A}$, DIV6 cortical neurons were labeled for TFII-I in red, TRPC3 in green, and NeuN in blue. Scale bar is shown in the first panel, WT TFII-I, 40 $\mu \mathrm{m}$. B, TFII-I, TRPC3, and NeuN fluorescence intensity was measured in the nucleus $\left(I_{N}\right)$, cytosol $\left(I_{C y t}\right)$ and plasma membrane $\left(I_{P m}\right)$. Fluorescence intensity was measured in a single section in the middle of the entire Z-stack for every neuron imaged. Scale bar, $5 \mu \mathrm{m}$. $\boldsymbol{C}, \mathrm{Src}^{\text {thl/thl }}$ mice had higher levels of TFII-I in the membrane than WT littermates. $\boldsymbol{D}$, There was no significant differential expression of TFII-I in the cytosol. $\boldsymbol{E}$, WT mice showed higher levels of TFII-I in the nucleus compared to Src ${ }^{\text {thl/thl }}$ littermates. $\boldsymbol{F}$, The $30 \%$ difference in TFII-I levels between the cytoplasm and nucleus in Src thl/thl mice and 17\% difference in WT mice shows that TFII-I was 
continued

more evenly distributed throughout the cell in WT mice. G, Src ${ }^{\text {thl/thl }}$ mice had higher levels of TRPC3 in the membrane than WT littermates. $\boldsymbol{H}$, Src ${ }^{\text {thl/thl }}$ mice had less TRPC3 in the cytosol than WT littermates. I, Src ${ }^{\text {thl/thl }}$ mice had less TRPC3 in the nucleus compared to WT littermates. $\boldsymbol{J}$, There was a 36\% difference in TRPC3 levels between the cytosol and the nucleus in Src ${ }^{\text {thl/thl }}$ mice. In WT mice, there was only a 17\% difference of fluorescence, which means that TRPC3 was more evenly distributed throughout the cell in WT mice. Data are expressed as mean \pm SEM, $* p<0.05$.

localization of TFII-I, resulting in altered protein levels in both the nucleus and the cell membrane.

Whilst in the cytoplasm, TFII-I regulates membrane localization of TRPC3 through binding to and sequestering of PLC- $\gamma$, which usually shuttles TRPC 3 subunits to the plasma membrane where they form a channel and allow agonist-inducible calcium entry (Caraveo et al, 2006). $\mathrm{Src}^{\text {thl/thl }}$ mice showed an increase in membrane-bound TRPC3 in whole-brain lysate $(p<0.05$; Fig. $9 B, C)$ and in primary cultured neurons (Fig. 10G), which mirrors the predicted state in WBS, where less TFII-I is available in the cell, resulting in more availability of PLC- $\gamma$.

Given that we found less TFII-I in the nucleus, and apparently unaltered levels of TFII-I in the cytosol (Fig. $10 A, D)$, the finding of more TRPC3 in the membrane is somewhat counterintuitive. However, the binding of TFII-I to PLC- $\gamma$ has itself been shown to be regulated by the phosphorylation of TFII-I on tyrosine residues 357 and 462 by Bruton's tyrosine kinase (BTK) in B cells (Egloff and Desiderio, 2001). This phosphorylation is proposed to alter the conformational state and allow competitive interaction with PLC- $\gamma$, but whether this process is carried out by an equivalent kinase in the brain remains unknown. In addition, Src itself has been shown to be an obligatory participant in TRPC3 channel activation, through an as yet unknown mechanism that does not involve either direct interaction or phosphorylation of TRPC3 (Vazquez et al, 2004).

Altered localization and/or regulation of TRPC3 is an attractive mechanism that could explain many of the features seen in the Src mutant mouse as well as in individuals with WBS. For example, TRPC3-mediated calcium currents are activated in the hippocampus in response to the release of activity-dependent growth factors ( $\mathrm{Li}$ et al, 2010), and the expression profile TRPC3 in the ear suggests it may contribute to development of cochlear tissues and play an important role in hair cell calcium homeostasis and regulation of auditory neurotransmission via G protein-coupled receptor signaling (Phan et al, 2010; Tadros et al, 2010). Investigation of the relationship between TFII-I, TRPC3, and agonist-induced calcium entry was initially established in B cells (Caraveo et al, 2006), but we have confirmed these findings in primary cortical neurons from mice that are heterozygously deleted or duplicated for Gtf2i (our unpublished observations). Reduced Src phosphorylation of TFII-I may also impact significantly on its function as a transcriptional activator in the nucleus since phosphorylation-deficient mutants failed to activate the c-fos promoter (Cheriyath et al, 2002).

Our results reveal a possible mechanistic link between Src signaling and the dual cellular roles of TFII-I. Recent analysis of a Gtf2i heterozygous deficient mouse also identified increased measures of social interaction, but no deficits in growth, craniofacial morphology, motor performance, startle response, or anxiety (Sakurai et al, 2011). Thus, we can conclude that an equivalent reduction of TFII-I activity alone cannot account for the varied phenotypes seen in the Src mutant mouse, nor in WBS. Disruption of TFII-I-mediated transcriptional activation and/or TRPC3-mediated calcium transport may, however, still underlie some of the cognitive and behavioral deficits in WBS individuals and Src mutant mice. A closely related protein, GTF2IRD1, is also deleted in WBS and has been linked to increased social interaction in mice, as well as deficits in cued fear conditioning, growth delay, hearing loss, and craniofacial abnormalities (Tassabehji et al, 2005; Young et al, 2008; Canales et al, 2014). It is possible that these two proteins both play a role in WBS, perhaps in a combinatorial manner, and that both are regulated by $\mathrm{Src}$ and have dual cellular functions. Deletion of the entire WBS syntenic region in mice does recapitulate many of the core features of WBS, including hypersoacibility, craniofacial abnormalities, reduced body weight, increased startle response, and impairments in motor coordination (Segura-Puimedon et al, 2014). Whether these symptoms are due primarily to the deletion of Gtf2i and Gtf2ird1, or whether additional genes are involved, remains to be determined.

In summary, we have identified a spectrum of neurobehavioral deficits in Src mutant mice, some of which overlap with the distinctive cognitive and behavioral profile of individuals with WBS. We have shown that $\mathrm{Src}^{\text {thl/thl }}$ mice have altered localization of TFII-I (coded for by a gene commonly deleted in WBS) and the TRPC3 calcium channel, which is regulated by both TFII-I and Src. This study establishes a link between Src signaling and WBS, a disorder with altered social behavior, and opens the possibility of targeting the Src pathway for therapeutic intervention in the future. Additional studies will be needed to determine the role of both Src and TFII-I in neuronal cells, and to understand how their disruption may contribute to neurodevelopmental disorders.

\section{REFERENCES}

Attias J, Raveh E, Ben-Naftali NF, Zarchi O, Gothelf D (2008) Hyperactive auditory efferent system and lack of acoustic reflexes in Williams syndrome. J Basic Clin Physiol Pharmacol 19:193-207. CrossRef

Axelsson S (2005) Variability of the cranial and dental phenotype in Williams syndrome. Swed Dent J Suppl 167:3-67.

Axelsson S, Kjaer I, Heiberg A, Bjørnland T, Storhaug K (2005) Neurocranial morphology and growth in Williams syndrome. Eur $\mathrm{J}$ Orthod 27:32-47. CrossRef Medline 
Berg JS, Brunetti-Pierri N, Peters SU, Kang SH, Fong CT, Salamone J, Freedenberg D, Hannig VL, Prock LA, Miller DT, Raffalli P, Harris DJ, Erickson RP, Cunniff C, Clark GD, Blazo MA, Peiffer DA, Gunderson KL, Sahoo T, Patel A, et al. (2007) Speech delay and autism spectrum behaviors are frequently associated with duplication of the 7q11.23 Williams-Beuren syndrome region. Genet Med 9:427-441. CrossRef Medline

Broeke JH, Roelandse M, Luteijn MJ, Boiko T, Matus A, Toonen RF, Verhage M (2010) Munc18 and Munc13 regulate early neurite outgrowth. Biol Cell 102:479-488. CrossRef Medline

Bromann PA, Korkaya H, Courtneidge SA (2004) The interplay between Src family kinases and receptor tyrosine kinases. Oncogene 23:7957-7968. CrossRef Medline

Canales CP, Wong AC, Gunning PW, Housely GD, Hardeman EC, Palmer SJ (2014) The role of GTF2IRD1 in the auditory pathology of Williams-Beuren syndrome. Eur J Hum Genet Sep 24 Epub ahead of print. CrossRef Medline

Caraveo G, van Rossum DB, Patterson RL, Snyder SH, Desiderio S (2006) Action of TFIl-I outside the nucleus as an inhibitor of agonist-induced calcium entry. Science 314:122-125. CrossRef Medline

Cheriyath V, Desgranges ZP, Roy AL (2002) c-Src-dependent transcriptional activation of TFII-I. J Biol Chem 277:22798-22805. CrossRef Medline

Doyle TF, Bellugi U, Korenberg JR, Graham J (2004) "Everybody in the world is my friend" hypersociability in young children with Williams syndrome. Am J Med Genet A 124A:263-273. CrossRef Medline

Egloff AM, Desiderio S (2001) Identification of phosphorylation sites for Bruton's tyrosine kinase within the transcriptional regulator BAP/TFII-I. J Biol Chem 276:27806-27815. CrossRef Medline

Enkhmandakh B, Makeyev AV, Erdenechimeg L, Ruddle FH, Chimge NO, Tussie-Luna MI, Roy AL, Bayarsaihan D (2009) Essential functions of the Williams-Beuren syndrome-associated TFII-I genes in embryonic development. Proc Natl Acad Sci U S A 106:181-186. CrossRef Medline

File SE, Cheeta S, Akanezi C (2001) Diazepam and nicotine increase social interaction in gerbils: a test for anxiolytic action. Brain Res 888:311-313. Medline

Gourbal BE, Barthelemy M, Petit G, Gabrion C (2004) Spectrographic analysis of the ultrasonic vocalisations of adult male and female BALB/c mice. Naturwissenschaften 91:381-385. CrossRef Medline

Hoffman F, Musolf K, Penn DJ (2012) Spectrographic analyses reveal signals of individuality and kinship in the ultrasonic courtship vocalizations of wild house mice. Physiol Behav 105:766-771. CrossRef Medline

Hoffman-Kim D, Kerner JA, Chen A, Xu A, Wang TF, Jay DG (2002) pp60(c-src) is a negative regulator of laminin-1-mediated neurite outgrowth in chick sensory neurons. Mol Cell Neurosci 21:81-93. Medline

Howard ML, Palmer SJ, Taylor KM, Arthurson GJ, Spitzer MW, Du X, Pang TY, Renoir T, Hardeman EC, Hannan AJ (2012) Mutation of Gtf2ird1 from the Williams-Beuren syndrome critical region results in facial dysplasia, motor dysfunction, and altered vocalisations. Neurobiol Dis 45:913-922. CrossRef Medline

Kiliaridis S, Engström C, Thilander B (1985) The relationship between masticatory function and craniofacial morphology. I. A cephalometric longitudinal analysis in the growing rat fed a soft diet. Eur $\mathrm{J}$ Orthod 7: 273-283 Medline

Kilkenny DM, Rocheleau JV, Price J, Reich MB, Miller GG (2003) c-Src regulation of fibroblast growth factor-induced proliferation in murine embryonic fibroblasts. J Biol Chem 278:17448-17454. CrossRef Medline

Kim H, Laing M, Muller W (2005) c-Src-null mice exhibit defects in normal mammary gland development and ERalpha signaling. Oncogene 24:5629-5636. CrossRef Medline

Kim JJ, Jung MW (2006) Neural circuits and mechanisms involved in Pavlovian fear conditioning: a critical review. Neurosci Biobehav Rev 30:188-202. CrossRef Medline
Kirshenbaum GS, Clapcote SJ, Duffy S, Burgess CR, Petersen J, Jarowek KJ, Yucel YH, Cortez MA, Snead OC 3rd, Vilsen B, Peever $\mathrm{JH}$, Ralph MR, Roder JC (2011) Mania-like behavior induced by genetic dysfunction of the neuron-specific $\mathrm{Na}+, \mathrm{K}+$-ATPase alpha3 sodium pump. Proc Natl Acad Sci U S A 108:18144-18149. CrossRef

Kuo SS, Armanini MP, Phillips HS, Caras IW (1997) Csk and BatK show opposite temporal expression in the rat CNS: consistent with its late expression in development, BatK induces differentiation of PC12 cells. Eur J Neurosci 9:2383-2393. Medline

Kuo WL, Chung KC, Rosner MR (1997) Differentiation of central nervous system neuronal cells by fibroblast-derived growth factor requires at least two signaling pathways: roles for Ras and Src. Mol Cell Biol 17:4633-4643.

Lahvis GP, Alleva E, Scattoni ML (2011) Translating mouse vocalizations: prosody and frequency modulation. Genes Brain Behav 10:4-16. CrossRef Medline

Levy D, Ronemus M, Yamrom B, Lee YH, Leotta A, Kendall J, Marks S, Lakshmi B, Pai D, Ye K, Buja A, Krieger A, Yoon S, Troge J, Rodgers L, lossifov I, Wigler M (2011) Rare de novo and transmitted copy-number variation in autistic spectrum disorders. Neuron 70:886-897. CrossRef Medline

Li Y, Calfa G, Inoue T, Amaral MD, Pozzo-Miller L (2010) Activitydependent release of endogenous BDNF from mossy fibers evokes a TRPC 3 current and $\mathrm{Ca} 2+$ elevations in CA3 pyramidal neurons. J Neurophysiol 103:2846-2856. CrossRef Medline

Lightfoot PS, German RZ (1998) The effects of muscular dystrophy on craniofacial growth in mice: a study of heterochrony and ontogenetic allometry. J Morphol 235:1-16. CrossRef Medline

Lowe C, Yoneda T, Boyce BF, Chen H, Mundy GR, Soriano P (1993) Osteopetrosis in Src-deficient mice is due to an autonomous defect of osteoclasts. Proc Natl Acad Sci U S A 90:4485-4489. Medline

Lu YM, Roder JC, Davidow J, Salter MW (1998) Src activation in the induction of long-term potentiation in CA1 hippocampal neurons. Science 279:1363-1367. Medline

Lucena J, Pezzi S, Aso E, Valero MC, Carreiro C, Dubus P, Sampaio A, Segura M, Barthelemy I, Zindel MY, Sousa N, Barbero JL, Maldonado R, Pérez-Jurado LA, Campuzano V (2010) Essential role of the N-terminal region of TFII-I in viability and behavior. BMC Med Genet 11:61. CrossRef Medline

Malenfant P, Liu X, Hudson ML, Qiao Y, Hrynchak M, Riendeau N, Hildebrand MJ, Cohen IL, Chudley AE, Forster-Gibson C, Mickelson EC, Rajcan-Separovic E, Lewis ME, Holden JJ (2012) Association of GTF2 $i$ in the Williams-Beuren syndrome critical region with autism spectrum disorders. J Autism Dev Disord 42:14591469. CrossRef Medline

Marshall CR, Noor A, Vincent JB, Lionel AC, Feuk L, Skaug J, Shago M, Moessner R, Pinto D, Ren Y, Thiruvahindrapduram B, Fiebig A, Schreiber S, Friedman J, Ketelaars CE, Vos YJ, Ficicioglu C, Kirkpatrick S, Nicolson R, Sloman L, et al. (2008) Structural variation of chromosomes in autism spectrum disorder. Am J Hum Genet 82:477-488. CrossRef Medline

Mclnnes LA, Nakamine A, Pilorge M, Brandt T, Jiménez González P, Fallas M, Manghi ER, Edelmann L, Glessner J, Hakonarson H, Betancur C, Buxbaum JD (2010) A large-scale survey of the novel $15 q 24$ microdeletion syndrome in autism spectrum disorders identifies an atypical deletion that narrows the critical region. Mol Autism 1:5. CrossRef Medline

Merla G, Brunetti-Pierri N, Micale L, Fusco C (2010) Copy number variants at Williams-Beuren syndrome $7 q 11.23$ region. Hum Genet 128:3-26. CrossRef Medline

Mervis CB (1999) The Williams syndrome cognitive profile: strengths, weaknesses, and interrelations among auditory short term memory, language, and visuospatial constructive cognition. In: Ecological approaches to cognition: essays in honor of Ulric Neisser (Winograd E, Fivush R, Hirst W, eds.), pp 193-227. Mahwah, NJ: Erlbaum

Mervis CB, John AE (2010) Cognitive and behavioral characteristics of children with Williams syndrome: implications for intervention 
approaches. Am J Med Genet C Semin Med Genet 154C:229-248. CrossRef Medline

Meyer-Lindenberg A, Kohn P, Mervis CB, Kippenhan JS, Olsen RK, Morris CA, Berman KF (2004) Neural basis of genetically determined visuospatial construction deficit in Williams syndrome. Neuron 43:623-631. CrossRef Medline

Moles A, Costantini F, Garbugino L, Zanettini C, D'Amato FR (2007) Ultrasonic vocalizations emitted during dyadic interactions in female mice: a possible index of sociability? Behav Brain Res 182: 223-230. CrossRef Medline

Nada S, Yagi T, Takeda H, Tokunaga T, Nakagawa H, Ikawa Y, Okada M, Aizawa S (1993) Constitutive activation of Src family kinases in mouse embryos that lack Csk. Cell 73:1125-1135. Medline

Nadler JJ, Moy SS, Dold G, Trang D, Simmons N, Perez A, Young NB, Barbaro RP, Piven J, Magnuson TR, Crawley JN (2004) Automated apparatus for quantitation of social approach behaviors in mice. Genes Brain Behav 3:303-314. CrossRef Medline

Ng D, Pitcher GM, Szilard RK, Sertie A, Kanisek M, Clapcote SJ, Lipina T, Kalia LV, Joo D, McKerlie C, Cortez M, Roder JC, Salter MW, Mclnnes RR (2009) Neto1 is a novel CUB-domain NMDA receptor-interacting protein required for synaptic plasticity and learning. PLoS Biol 7:e41. CrossRef Medline

Ng R, Jarvinen A, Bellugi U (2014) Toward a deeper characterization of the social phenotype of Williams syndrome: The association between personality and social drive. Res Dev Disabil 35:18381849. CrossRef Medline

Ohazama A, Sharpe PT (2007) TFII-I gene family during tooth development: candidate genes for tooth anomalies in Williams syndrome. Dev Dyn 236:2884-2888. CrossRef Medline

Okada M, Nada S, Yamanashi Y, Yamamoto T, Nakagawa H (1991) CSK: a protein-tyrosine kinase involved in regulation of src family kinases. J Biol Chem 266:24249-24252. Medline

Phan PA, Tadros SF, Kim Y, Birnbaumer L, Housley GD (2010) Developmental regulation of TRPC3 ion channel expression in the mouse cochlea. Histochem Cell Biol 133:437-448. CrossRef Medline

Phelps EA, LeDoux JE (2005) Contributions of the amygdala to emotion processing: from animal models to human behavior. Neuron 48:175-187. CrossRef Medline

Roy AL, Meisterernst M, Pognonec P, Roeder RG (1991) Cooperative interaction of an initiator-binding transcription initiation factor and the helix-loop-helix activator USF. Nature 354:245-248. CrossRef Medline

Sakurai T, Dorr NP, Takahashi N, Mclnnes LA, Elder GA, Buxbaum JD (2011) Haploinsufficiency of Gtf2i, a gene deleted in Williams Syndrome, leads to increases in social interactions. Autism Res 4:28-39. CrossRef Medline

Sanders SJ, Ercan-Sencicek AG, Hus V, Luo R, Murtha MT, MorenoDe-Luca D, Chu SH, Moreau MP, Gupta AR, Thomson SA, Mason CE, Bilguvar K, Celestino-Soper PB, Choi M, Crawford EL, Davis L, Wright NR, Dhodapkar RM, DiCola M, DiLullo NM, et al. (2011) Multiple recurrent de novo CNVs, including duplications of the $7 q 11.23$ Williams syndrome region, are strongly associated with autism. Neuron 70:863-885. CrossRef Medline

Segura-Puimedon M, Sahún I, Velot E, Dubus P, Borralleras C, Rodrigues AJ, Valero MC, Valverde O, Sousa N, Herault Y, Dierssen M, Pérez-Jurado LA, Campuzano V (2014) Heterozygous deletion of the Williams-Beuren syndrome critical interval in mice recapitulates most features of the human disorder. Hum Mol Genet 23:6481-6494. CrossRef Medline

Shahbazian M, Young J, Yuva-Paylor L, Spencer C, Antalffy B, Noebels J, Armstrong D, Paylor R, Zoghbi H (2002) Mice with truncated MeCP2 recapitulate many Rett syndrome features and display hyperacetylation of histone H3. Neuron 35:243-254. Medline

Sinai L, Duffy S, Roder JC (2010) Src inhibition reduces NR2B surface expression and synaptic plasticity in the amygdala. Learn Mem 17:364-371. CrossRef Medline

Soriano P, Montgomery C, Geske R, Bradley A (1991) Targeted disruption of the c-src proto-oncogene leads to osteopetrosis in mice. Cell 64:693-702. Medline

Spencer CM, Alekseyenko O, Serysheva E, Yuva-Paylor LA, Paylor R (2005) Altered anxiety-related and social behaviors in the Fmr1 knockout mouse model of fragile X syndrome. Genes Brain Behav 4:420-430. CrossRef Medline

Tadros SF, Kim Y, Phan PA, Birnbaumer L, Housley GD (2010) TRPC3 ion channel subunit immunolocalization in the cochlea. Histochem Cell Biol 133:137-147. CrossRef Medline

Tassabehji M, Hammond P, Karmiloff-Smith A, Thompson P, Thorgeirsson SS, Durkin ME, Popescu NC, Hutton T, Metcalfe K, Rucka A, Stewart H, Read AP, Maconochie M, Donnai D (2005) GTF2IRD1 in craniofacial development of humans and mice. Science 310:1184-1187. CrossRef Medline

Thomas SM, Brugge JS (1997) Cellular functions regulated by Src family kinases. Annu Rev Cell Dev Biol 13:513-609. CrossRef Medline

Tiffee JC, Xing L, Nilsson S, Boyce BF (1999) Dental abnormalities associated with failure of tooth eruption in src knockout and op/op mice. Calcif Tissue Int 65:53-58. Medline

Van der Aa N, Rooms L, Vandeweyer G, van den Ende J, Reyniers E, Fichera M, Romano C, Delle Chiaie B, Mortier G, Menten B, Destrée A, Maystadt I, Männik K, Kurg A, Reimand T, McMullan D, Oley C, Brueton L, Bongers EM, van Bon BW, et al. (2009) Fourteen new cases contribute to the characterization of the $7 q 11.23$ microduplication syndrome. Eur J Med Genet 52:94-100. CrossRef Medline

Vazquez G, Wedel BJ, Kawasaki BT, Bird GS, Putney JW Jr (2004) Obligatory role of Src kinase in the signaling mechanism for TRPC3 cation channels. J Biol Chem 279:40521-40528. CrossRef Medline

Wang H, Liang S, Burgdorf J, Wess J, Yeomans J (2008) Ultrasonic vocalizations induced by sex and amphetamine in M2, M4, M5 muscarinic and D2 dopamine receptor knockout mice. PLoS One 3:e1893. CrossRef Medline

Wang YT, Salter MW (1994) Regulation of NMDA receptors by tyrosine kinases and phosphatases. Nature 369:233-235. CrossRef Medline

Wrenn CC, Harris AP, Saavedra MC, Crawley JN (2003) Social transmission of food preference in mice: methodology and application to galanin-overexpressing transgenic mice. Behav Neurosci 117:21-31. CrossRef Medline

Young EJ, Lipina T, Tam E, Mandel A, Clapcote SJ, Bechard AR, Chambers J, Mount HT, Fletcher PJ, Roder JC, Osborne LR (2008) Reduced fear and aggression and altered serotonin metabolism in Gtf2ird1-targeted mice. Genes Brain Behav 7:224-234. CrossRef Medline

Zampieri BL, Fernandez F, Pearson JN, Stasko MR, Costa AC (2014) Ultrasonic vocalizations during male-female interaction in the mouse model of Down syndrome Ts65Dn. Physiol Behav 128:119125. CrossRef Medline

Zhang S, Edelmann L, Liu J, Crandall JE, Morabito MA (2008) Cdk5 regulates the phosphorylation of tyrosine 1472 NR2B and the surface expression of NMDA receptors. J Neurosci 28:415-424. CrossRef Medline 